\title{
Space-Frequency Block Code with Matched Rotation for MIMO-OFDM System with Limited Feedback
}

\author{
Min Zhang, ${ }^{1}$ Thushara D. Abhayapala, ${ }^{1}$ Dhammika Jayalath, ${ }^{2}$ \\ David Smith, ${ }^{3}$ and Chandra Athaudage ${ }^{4}$ \\ ${ }^{1}$ College of Engineering \& Computer Science, Australian National University, Canberra, ACT 0200, Australia \\ ${ }^{2}$ Faculty of Built Environment \& Engineering, Queensland University of Technology, Brisbane, QLD 4001, Australia \\ ${ }^{3}$ National ICT Australia Limited, Canberra, ACT 2601, Australia \\ ${ }^{4}$ Department of Electrical \& Electronic Engineering, University of Melbourne, Melbourne, VIC 301, Australia
}

Correspondence should be addressed to Thushara D. Abhayapala, thushara.abhayapala@anu.edu.au

Received 30 November 2008; Revised 19 April 2009; Accepted 24 June 2009

Recommended by Markus Rupp

\begin{abstract}
This paper presents a novel matched rotation precoding (MRP) scheme to design a rate one space-frequency block code (SFBC) and a multirate SFBC for MIMO-OFDM systems with limited feedback. The proposed rate one MRP and multirate MRP can always achieve full transmit diversity and optimal system performance for arbitrary number of antennas, subcarrier intervals, and subcarrier groupings, with limited channel knowledge required by the transmit antennas. The optimization process of the rate one MRP is simple and easily visualized so that the optimal rotation angle can be derived explicitly, or even intuitively for some cases. The multirate MRP has a complex optimization process, but it has a better spectral efficiency and provides a relatively smooth balance between system performance and transmission rate. Simulations show that the proposed SFBC with MRP can overcome the diversity loss for specific propagation scenarios, always improve the system performance, and demonstrate flexible performance with large performance gain. Therefore the proposed SFBCs with MRP demonstrate flexibility and feasibility so that it is more suitable for a practical MIMO-OFDM system with dynamic parameters.
\end{abstract}

Copyright (C) 2009 Min Zhang et al. This is an open access article distributed under the Creative Commons Attribution License, which permits unrestricted use, distribution, and reproduction in any medium, provided the original work is properly cited.

\section{Introduction}

A multiple-input multiple-output (MIMO) communication system has an increased spectral efficiency in a wireless channel. It can provide both high rate transmission and spatial diversity between any transmit-receive pair. The appropriate space time block code (STBC) allows us to achieve, or approach, channel capacity for the flat fading propagation channel with multiple antennas [1-4]. Moreover, an orthogonal frequency division multiplexing (OFDM) system transforms a frequency selective fading channel into a number of parallel subsystems with flat fading. It can eliminate the inter symbol interference (ISI) completely by inserting a long enough cyclic prefix (CP). The MIMO-OFDM system has attracted much attention for future broadband wireless systems and has already been implemented in IEEE802.11n, WiMax [5] and 3G-LTE systems $[6,7]$.
For MIMO-OFDM systems, various space-time/ frequency codes have been developed to achieve spatial, multipath, and temporal diversities by coding across multiple antennas, subcarriers, and OFDM symbol intervals [8]. All existing STBCs, for example, $[1,9,10]$, can be converted into space-frequency block codes (SFBCs) simply by spreading the time domain signal of STBC within the frequency domain. This conversion works well if adjacent subcarrier channels are highly correlated, for example, Alamouti code [1] proposed to be deployed within the LTE system [6]. However this kind of direct conversion [11] is not optimal and fails to achieve valuable frequency diversity that can improve system performance.

A SFBC should be able to achieve both spatial and frequency diversity. The SFBCs proposed in [12-14] achieve full spatial and frequency (multipath) diversities by coding across multiple antennas and subcarriers. These SFBCs require at least $N_{t}(L+1)$ subcarriers to achieve full diversity 
order where $L$ is the fixed channel order (the number of paths) and $N_{t}$ is the number of transmit antennas. The channel order provides an upperbound in the rank of the frequency correlation matrix of the OFDM system [15]. Hence by employing more than a threshold number of subcarriers, full spatial and frequency diversities can be achieved. However the channel order $L$ might be large, for example, $L+1=20$ in [16], and vary with users and scatterer movement, raising questions about the practical implementation of these SFBCs.

On the other hand, the design of SFBC provides a fundamental understanding so that a variety of space-timefrequency block codes (STFBCs) are proposed for particular system requirements and channel conditions. Essentially these STFBCs do not differ significantly from either SFBC or STBC. Some STFBCs have assumed that consecutive OFDM intervals are static during a period of time. For example, a rate one STFBC is proposed in [17] by combining orthogonal STBC [18] and linear dispersion codes $[9,19]$, and also proposed in [20,21] using quasiorthogonal block codes [22]. Alternatively some STFBCs have assumed that consecutive OFDM intervals are independent (or slightly correlated) during a period of time so that temporal diversity could be achieved. For example, the rate one STFBC proposed in [23] extends SFBC in [13] into all space, time, and frequency domains. High rate full diversity STFBCs are proposed in $[24,25]$ using a layered algebraic design.

The SFBC proposed in $[12,23]$ does not require knowledge of the channel power delay profile (PDP) at the transmit end. However it is verified only for specific channel conditions and provides an upperbound of performance so that the diversity lose may happen. To overcome this problem and also optimize the system performance, perfect knowledge of channel PDP is required by the transmit antennas in the optimization process proposed in [13] and further high rate SFBC design proposed in $[24,25]$. Such an assumption might not be feasible for a practical implementation. Moreover, the optimization process proposed in [13] adjusted the subcarrier interval to improve the performance. But the optimal subcarrier interval might not be a factor of $N_{c}$ where $N_{c}$ is the number of subcarriers of a MIMO-OFDM system. Hence partial subcarriers of the system cannot achieve such optimal subcarrier interval after grouping. Furthermore, a MIMO-OFDM system is usually divided into a number of MIMO-OFDM subsystems by subcarrier grouping. In a multiuser scenario each user will be allocated one or more subsystems. This property leads to diverse optimal subcarrier intervals for different subsystems and users. Then a new problem of subcarrier grouping is raised since all users in the system will compete with each other to get a better allocation of subcarriers.

Because of relatively large channel order in real propagation scenarios, achieving full space and frequency diversity is not a top priority but how to achieve a given transmit diversity order efficiently across both space and frequency domains is a more important question. Moreover, considering the difficulty in realization of full knowledge of channel PDP at the transmit end, and the limitation of optimization for subcarrier interval, a novel matched rotation precoding
(MRP) is proposed in this paper. At first, the basic structure and design criteria of SFBC demonstrate the repetition and rotation patterns, which do not exist in the traditional STBC design. Moreover, the proposed SFBC design structure focuses on the scenario of partial knowledge of channel PDP known by the transmit antennas through the link feedback. Then a rate one MRP and a multirate MRP are proposed, both of which are capable of achieving full transmit diversity for the MIMO-OFDM system with an arbitrary number of antennas, subcarrier interval, or subcarrier grouping. The rate one MRP has a relatively simple optimization process, which can be transformed into an explicit diagram. The optimal rotation angles of MRP can be derived explicitly, or even intuitively in some cases. On the other hand, the multirate MRP has a more complex optimization process but has better spectral efficiency than the rate one MRP. Hence a better performance can be achieved by the multirate MRP if the same bit transmission rate is assumed. It is also capable of achieving a relatively smooth balance between system performance and transmission rate without significantly changing the coding structure.

The rest of the paper is organized as follows. Section 2 describes a model for the MIMO-OFDM system and reviews the correlation structure between space and frequency domains. Section 3 presents design criteria of SFBC and reveals the distinct repetition and rotation patterns. Design structures for scenarios with full or limited knowledge of PDP are also compared and investigated in this section. Then Section 4 introduces a rate one MRP with limited feedback knowledge and corresponding optimization process. And Section 5 introduces a multirate MRP with limited feedback knowledge and corresponding optimization process. Section 6 provides simulation results, and Section 7 concludes the paper.

Notation 1. Matrices and vectors are denoted by boldface letters. The $(\cdot)^{T},(\cdot)^{*}$, and $(\cdot)^{\dagger}$ are defined as matrix transpose, complex conjugate, and adjoint of complex conjugate transpose, respectively. The process of "vec" is defined as a matrix reconstruction which stacks a matrix columnwise to form a column vector. $\otimes$ and $\circ$ are defined as Kronecker product and Hadamard product, respectively. $\mathbf{1}_{a}$ and $\mathbf{1}_{a \times b}$ are defined as $a \times a$ and $a \times b$ all one matrices, respectively. $\mathbf{I}_{a}$ is defined as an $a \times a$ identity matrix.

\section{MIMO-OFDM System Modelling}

This section presents a general MIMO-OFDM system model and proposes a concise SFBC design structure that is used to design precoding matrices and to optimize coding gain and diversity gain. The MIMO-OFDM system model is simplified with some preliminary assumptions, compared with complex SCM model [26] or WINNER model [16]. It is assumed that the MIMO-OFDM system model has perfect synchronization between transmit and receive antennas, and also among the users so that the system has no ISI. The AoA and AoD of the MIMO channels are assumed to be uncorrelated. 
2.1. Subcarrier Grouping for the MIMO-OFDM Model. We consider a MIMO-OFDM system with $N_{t}$ transmit antennas, $N_{r}$ receive antennas and $N_{c}$ subcarriers. The frequency selective channel is assumed to be static (timeinvariant) within at least one OFDM symbol interval $T_{s}$. Each transmit and receive pair has $L+1$ resolvable delay paths with the same PDP, for example, SCM [26] and COST207 [27]. A block of data symbols is transmitted over each transmit antenna and passed through a $N_{c}$-point inverse fast Fourier transform and followed by the appending of a CP. The length of CP is chosen to be long enough to remove the ISI completely. At each receive antenna the $\mathrm{CP}$ is removed at first and then a fast Fourier transform is applied. Hence the MIMO frequency selective fading channel is decoupled into $N_{c}$ parallel MIMO flat fading channels.

To reduce system complexity while preserving both diversity and coding gain, a MIMO-OFDM system typically is partitioned into $N_{s}$ MIMO-OFDM subsystems where $N_{s} \geq 1$. It is pointed out in [28] that the MIMOOFDM system capacity with grouping can approach the channel capacity without grouping very closely. Hence the performance of the system is evaluated by the averaged performance of all subsystems. Here we consider a subsystem with $P$ subcarriers selected from a total of $N_{c}$ subcarriers where $P$ is an arbitrary integer greater than $N_{t}$. The subcarriers in the subsystem are equally separated from each other with a positive integer interval $\delta$. The optimization process by tuning subcarrier interval $\delta$ was proposed in [13]. However due to the limitations of implementation, the subcarrier interval $\delta$ is fixed in a MIMO-OFDM subsystem in this paper. Therefore, it is assumed that $\delta=\left\lfloor N_{c} / P\right\rfloor$ where $\lfloor a\rfloor$ denotes the largest integer less than or equal to $a$ so that the subcarriers are separated as far as they can be in the subsystem. The rest of $\left(N_{c}-\right.$ $\delta P)<P$ subcarriers could be used as guard intervals to separate OFDM symbols. Then a MIMO-OFDM system is partitioned into $N_{s}=\delta$ MIMO-OFDM subsystems who preserve exactly same second order characteristics. Hence the proposed SFBC design only focuses on an arbitrary MIMO-OFDM subsystem. For a multiuser scenario, each user can be allocated one or more MIMO-OFDM subsystems depending on the system complexity and requirement. The block diagram of a MIMO-OFDM system is shown in Figure 1.

The channel frequency response $h_{m n}(p)$ over the $p$ th subcarrier in the MIMO-OFDM subsystem between transmit antenna $m$ where $\left(m \in\left[1, \ldots, N_{t}\right]\right)$ and receive antenna $n$ where $\left(n \in\left[1, \ldots, N_{r}\right]\right)$ is given by

$$
h_{m n}(p)=\sum_{\ell=0}^{L} \hbar_{m n, \ell} e^{-j 2 \pi((p-1) \delta+1) \tau_{\ell} / T_{s}},
$$

where $p \in[1, \ldots, P]$ and $\ell \in[0, \ldots, L], \tau_{\ell}$ and $\hbar_{m n, \ell}$ are the delay and complex amplitude coefficient of the $\ell$ th path, respectively, and $T_{s}$ is the OFDM symbol interval. The channel frequency response between transmit and receive antennas for the $p$ th subcarrier in the MIMO-OFDM subsystem is denoted by

$$
\mathbf{H}(p)=\left[\begin{array}{ccc}
h_{11}(p) & \cdots & h_{1 N_{r}}(p) \\
\vdots & \cdots & \vdots \\
h_{N_{t} 1}(p) & \cdots & h_{N_{t} N_{r}}(p)
\end{array}\right],
$$

where each entry $h_{m n}(p)$ is given by (1). Then the $P N_{t} \times N_{r}$ channel matrix $\widetilde{\mathbf{H}}$ is constructed by stacking up these channel matrices $\mathbf{H}(p)$ columnwisely and shown as

$$
\widetilde{\mathbf{H}}=\left[\mathbf{H}(1)^{T}, \ldots, \mathbf{H}(P)^{T}\right]^{T} .
$$

Suppose that the transmitted symbol vector $\mathbf{S}$ is defined as $\mathbf{S}=\left[s_{1,1}, \ldots, s_{1, N_{t}}, \ldots, s_{P, 1}, \ldots, s_{P, N_{t}}\right]$ where two subscripts denote specific subcarrier and transmit antenna, respectively. Moreover, the transmission power of vector $\mathbf{S}$ is normalized within each SFBC design and each MIMO-OFDM subsystem. It is given by $\mathbb{E}\left[\mathbf{S S}^{\dagger}\right]=P$. Hence the receive signal of each subsystem, a $P N_{r} \times 1$ vector $\mathrm{Y}$, can be expressed as

$$
\mathbf{Y}=\sqrt{\frac{\rho}{N_{t}}} \widehat{\mathbf{S}}_{\operatorname{vec}}(\tilde{\mathbf{H}})+\mathbf{Z},
$$

where $\hat{\mathbf{S}}=\left\{\left(\mathbf{I}_{N_{r} P} \otimes \mathbf{1}_{1 \times N_{t}}\right) \circ\left(\mathbf{1}_{N_{r} P \times N_{r}} \otimes \mathbf{S}\right)\right\}$. The channel state information $\widetilde{\mathbf{H}}$ is assumed to be perfectly known at the receive end, but not known at the transmit end. $\rho$ is the average signal to noise ratio (SNR) at each receive antenna, independent of the number of transmit antennas and receive antennas. The noise vector $\mathbf{Z}$ is assumed to be additive white Gaussian noise with zero mean and unit variance.

\subsection{Correlation Structure of the MIMO-OFDM Subsystem.} The MIMO-OFDM subsystem is assumed to have arbitrary spatial correlation structures at both transmit and receive ends. The spatial correlation matrix between two ends is separable because of independent outgoing and incoming propagation $[29,30]$. Furthermore, with the assumption that the space, time, and frequency domains are independent of each other [13], the correlation coefficient between the channel frequency response $h_{m n}(p)$ and $h_{m^{\prime} n^{\prime}}\left(p^{\prime}\right)$ is given by

$$
\mathbb{E}\left\{h_{m n}(p) h_{m^{\prime} n^{\prime}}^{*}\left(p^{\prime}\right)\right\}=\mathbf{R}_{B S}\left(m, m^{\prime}\right) \mathbf{R}_{M S}\left(n, n^{\prime}\right) \mathbf{R}_{F}\left(p, p^{\prime}\right),
$$

where scalars $\mathbf{R}_{B S}\left(m, m^{\prime}\right), \mathbf{R}_{M S}\left(n, n^{\prime}\right)$, and $\mathbf{R}_{F}\left(p, p^{\prime}\right)$ are transmit spatial, receive spatial, and frequency correlation coefficients respectively. They are defined as

$$
\begin{aligned}
\mathbf{R}_{B S}\left(m, m^{\prime}\right) & =\mathbb{E}\left\{h_{m n}(p) h_{m \prime n}^{*}(p)\right\}, \\
\mathbf{R}_{M S}\left(n, n^{\prime}\right) & =\mathbb{E}\left\{h_{m n}(p) h_{m n^{\prime}}^{*}(p)\right\}, \\
\mathbf{R}_{F}\left(p, p^{\prime}\right) & =\mathbb{E}\left\{h_{m n}(p) h_{m n}^{*}\left(p^{\prime}\right)\right\}=\mathbf{w}_{p} \mathbf{R}_{D} \mathbf{w}_{p^{\prime}}^{\dagger}, \\
\mathbf{R}_{D}\left(\ell, \ell^{\prime}\right) & =\mathbb{E}\left\{\hbar_{m n, \ell} \hbar_{m n, \ell^{\prime}}^{*}\right\} .
\end{aligned}
$$

Furthermore, the frequency correlation matrix $\mathbf{R}_{F}$ is given by

$$
\mathbf{R}_{F}=\mathbf{W R}_{D} \mathbf{W}^{\dagger} .
$$




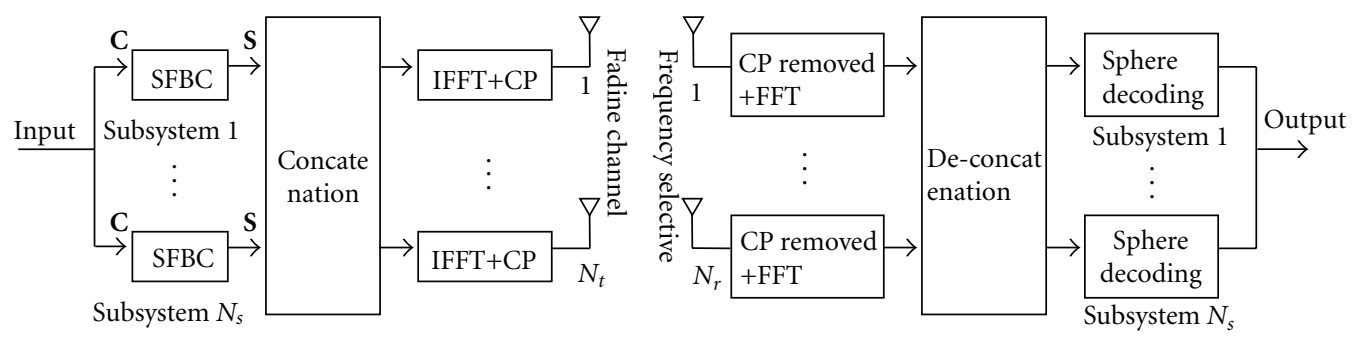

FIgURE 1: SFBC block diagram for a MIMO-OFDM system.

The $P \times(L+1)$ matrix $\mathbf{W}$ is shown as

$$
\mathbf{W}=\left[\mathbf{w}^{0}, \ldots, \mathbf{w}^{L}\right]=\left[\begin{array}{c}
\mathbf{w}_{1} \\
\vdots \\
\mathbf{w}_{P}
\end{array}\right]=\left[\begin{array}{ccc}
1 & \cdots & 1 \\
\vdots & \cdots & \vdots \\
w_{P}^{0} & \cdots & w_{P}^{L}
\end{array}\right],
$$

where the entry $w_{p}^{\ell}$ in matrix $\mathbf{W}$ is defined as $w_{p}^{\ell}=$ $e^{j 2 \pi(p-1) \delta \tau_{\ell} / T_{s}}$. Moreover, the MIMO-OFDM subsystem has an underlying assumption of $2 \pi \delta \tau_{\ell} / T_{s} \neq 2 k \pi+2 \pi \delta \tau_{\ell^{\prime}} / T_{s}$ for $\forall \ell \neq \ell^{\prime}, \ell, \ell^{\prime} \in[0, \ldots, L]$ and $k \in \mathbb{Z}$. Otherwise the MIMOOFDM subsystem will suffer the loss of diversity gain.

Therefore, we have

$$
\mathbb{E}\left[\operatorname{vec}(\tilde{\mathbf{H}}) \operatorname{vec}^{\dagger}(\tilde{\mathbf{H}})\right]=\mathbf{R}_{M S} \otimes \mathbf{R}_{F} \otimes \mathbf{R}_{B S}
$$

where entries of correlation matrices $\mathbf{R}_{M S}, \mathbf{R}_{F}$, and $\mathbf{R}_{B S}$ are given by (6).

\section{Analysis of SFBC Design}

In this section the basic design criteria of SFBC are reviewed and distinct rotation/repetition patterns are revealed to show the specialty of SFBC.

3.1. Design Criteria. The average pairwise error probability (PEP) between the codeword $\mathbf{C}$ and $\widetilde{\mathbf{C}}$ over all channel realizations can be upper bounded by [31]

$$
P(\mathbf{C} \longrightarrow \tilde{\mathbf{C}}) \leq\left(\frac{\rho}{4 N_{t}}\right)^{-\operatorname{rank}(\boldsymbol{\Lambda})}\left(\prod_{i=1}^{\operatorname{rank}(\boldsymbol{\Lambda})} \lambda_{i}(\boldsymbol{\Lambda})\right)^{-1}
$$

where rank $(\boldsymbol{\Lambda})$ and $\lambda_{i}(\boldsymbol{\Lambda})$ are the rank and the $i$ th nonzero eigenvalue of the covariance matrix $\Lambda$, respectively. The matrix $\Lambda$ is further given by

$$
\begin{aligned}
\boldsymbol{\Lambda} & =\mathbb{E}\left[\Delta \widehat{\mathbf{S}} \operatorname{vec}(\tilde{\mathbf{H}}) \operatorname{vec}^{\dagger}(\tilde{\mathbf{H}}) \Delta \hat{\mathbf{S}}^{\dagger}\right] \\
& =\Delta \widehat{\mathbf{S}}\left\{\mathbf{R}_{M S} \otimes \mathbf{R}_{F} \otimes \mathbf{R}_{B S}\right\} \Delta \hat{\mathbf{S}}^{\dagger} \\
& =\mathbf{R}_{M S} \otimes\left\{\left(\Delta \widetilde{\mathbf{S}} \mathbf{R}_{B S} \Delta \tilde{\mathbf{S}}^{\dagger}\right) \circ \mathbf{R}_{F}\right\},
\end{aligned}
$$

where the $P \times N_{t}$ matrix $\Delta \widetilde{\mathbf{S}}$ is stacked up from $\Delta \mathbf{S}$ and given by

$$
\begin{aligned}
\Delta \widetilde{\mathbf{S}}^{m} & =\left[\left(\Delta s_{1, m}, \ldots, \Delta s_{P, m}\right)\right]^{T}, \\
\Delta \widetilde{\mathbf{S}} & =\left[\Delta \widetilde{\mathbf{S}}^{1}, \ldots, \Delta \widetilde{\mathbf{S}}^{N_{t}}\right] .
\end{aligned}
$$

Each row vector of $\Delta \tilde{\mathbf{S}}$ is transmitted by $N_{\mathbf{t}}$ transmit antennas through the same subcarrier, and each column vector is transmitted by $P$ subcarriers through the same transmit antenna. Hence to improve system performance, both coding gain and diversity gain should be optimized by carefully designing $\left(\Delta \widetilde{\mathbf{S}} \mathbf{R}_{B S} \Delta \widetilde{\mathbf{S}}^{\dagger}\right) \circ \mathbf{R}_{F}$, but both gains are independent of receive spatial correlation.

For instance, if $\mathbf{R}_{F} \approx \mathbf{1}_{P}$, for example, when the subcarrier interval $\delta=1$ and the value of $N_{c}$ is relatively large, the design of SFBC has no difference with traditional STBC in which the coding gain is optimized by a subsequent structure of $\Delta \widetilde{\mathbf{S}} \mathbf{R}_{B S} \Delta \widetilde{\mathbf{S}}$. If these $P$ subcarriers are independent from each other [17], then $\mathbf{R}_{F}=\mathbf{I}_{P}$. The design criterion is simplified as maximizing $\prod_{p=1}^{P}\left(\sum_{m=1}^{N_{t}}\left\|\Delta s_{p, m}\right\|^{2}\right)$. It has a simple lowerbound, $N_{t}^{P}\left(\prod_{p=1}^{P} \prod_{m=1}^{N_{t}}\left\|\Delta s_{p, m}\right\|\right)^{2 / N_{t}}$ which could be optimized by linear dispersion codes [32].

3.2. Structure Analysis with Full Knowledge of PDP. Some further assumptions are descripted in this section. It is assumed that the knowledge of channel PDP is fed back to the transmit antennas through uplink transmission or data feedback. Therefore time delays $\tau_{\ell}$ and corresponding delay power $\sigma_{\ell}^{2}$ are perfectly known at the transmit end. And at the same time the receive end knows the channel state information $\widetilde{\mathbf{H}}$ perfectly for the decoding process. The SFBC design with limited knowledge of PDP will be discussed next and compared with the scenario of full knowledge of channel PDP.

The channel between the $m$ th transmit antenna and the $n$th receive antenna experiences frequency-selective fading induced by $L+1$ independent wireless propagation paths. The coefficient $\hbar_{m n, \ell}$ is assumed to be an uncorrelated circularly symmetric complex Gaussian random variable with zero mean and variance $\sigma_{\ell}^{2}$ given by the channel PDP, which is sorted in a decreasing order so as to $\sigma_{0}^{2} \geq \cdots \geq \sigma_{L}^{2}$. Hence we have $\mathbf{R}_{B S}=\mathbf{I}_{N_{t}}$ and $\mathbf{R}_{M S}=\mathbf{I}_{N_{r}}$. Furthermore, the matrix $\mathbf{R}_{D}$ is a diagonal matrix given by $\mathbf{R}_{D}(\ell, \ell)=\sigma_{\ell}^{2}$ and $\sum_{\ell=0}^{L} \sigma_{\ell}^{2}=1$. The number of subcarriers in the MIMO-OFDM subsystem is assumed to be $P \leq N_{t}(L+1)$ and $P>N_{t}$. Therefore equation (11) shows that the maximal achievable transmit diversity is $P$.

By utilizing these assumptions and definitions, the covariance matrix $\Lambda$ in (11) is given by

$$
\boldsymbol{\Lambda}=\mathbf{I}_{N_{r}} \otimes\left\{\left(\Delta \widetilde{\mathbf{S}} \Delta \tilde{\mathbf{S}}^{\dagger}\right) \circ\left(\mathbf{W R}_{D} \mathbf{W}^{\dagger}\right)\right\}
$$


Therefore if the covariance matrix $\Lambda$ has full rank, the determinant of $\Lambda$ is given by the following.

(1) If $P=N_{t}(L+1)$ (full spatial and frequency diversity as achieved in [13]), or $\mathbf{R}_{D}=(1 /(L+1)) \mathbf{I}_{L+1}$ (uniform PDP as adopted in [12]), we have

$$
\operatorname{det}(\boldsymbol{\Lambda})=\left(\prod_{\ell=0}^{L} \sigma_{\ell}^{2}\right)^{N_{t} N_{r}}\|\operatorname{det}(\boldsymbol{\Omega})\|^{2 \mathrm{~N}_{\mathrm{r}}},
$$

where $\boldsymbol{\Omega}$ is a $P \times N_{t}(L+1)$ complex square matrix and reconstructed as

$$
\begin{gathered}
\boldsymbol{\Omega}=\left[\left(\Delta \widetilde{\mathbf{S}}^{1} \circ \mathbf{w}^{0}\right), \ldots,\left(\Delta \widetilde{\mathbf{S}}^{N_{t}} \circ \mathbf{w}^{0}\right), \ldots,\right. \\
\left.\left(\Delta \widetilde{\mathbf{S}}^{1} \circ \mathbf{w}^{L}\right), \ldots,\left(\Delta \widetilde{\mathbf{S}}^{N_{t}} \circ \mathbf{w}^{L}\right)\right],
\end{gathered}
$$

where $\Delta \widetilde{\mathbf{S}}^{m}$ is the $m$ th column vector from matrix $\Delta \widetilde{\mathbf{S}}$

(2) If $N_{t}<P<N_{t}(L+1)$ and $\mathbf{R}_{D}$ is not an identity matrix, we have

$$
\operatorname{det}(\boldsymbol{\Lambda})=\left\{\operatorname{det}\left(\boldsymbol{\Omega} \boldsymbol{\Omega}^{\dagger}\right)\right\}^{N_{r}},
$$

where $\Omega$ is a $P \times N_{t}(L+1)$ complex matrix that is reconstructed as

$$
\begin{aligned}
\boldsymbol{\Omega}= & {\left[\left(\sigma_{0} \Delta \widetilde{\mathbf{S}}^{1} \circ \mathbf{w}^{0}\right), \ldots,\left(\sigma_{0} \Delta \widetilde{\mathbf{S}}^{N_{t}} \circ \mathbf{w}^{0}\right), \ldots,\right.} \\
& \left.\left(\sigma_{L} \Delta \widetilde{\mathbf{S}}^{1} \circ \mathbf{w}^{L}\right), \ldots,\left(\sigma_{L} \Delta \widetilde{\mathbf{S}}^{N_{t}} \circ \mathbf{w}^{L}\right)\right] .
\end{aligned}
$$

Remark 1. Equations (14) and (16) show that the design of SFBC is separable from the delay power $\sigma_{\ell}$ only if $P=$ $N_{t}(L+1)$ or $\mathbf{R}_{D}$ is an identity matrix. Hence two types of matrix $\boldsymbol{\Omega}$ are given in (14) and (16) separately. The matrix $\Omega$ in (14) is independent of $\sigma_{\ell}$, and more generally the matrix $\Omega$ in (16) is embedded with $\sigma_{\ell}$. Moreover, the matrix $\Omega$ reveals the characteristics of repetition and rotation patterns of the SFBC which do not exist in the traditional STBC design. The matrix $\boldsymbol{\Omega}$ is a pattern of $\Delta \widetilde{\mathbf{S}}$ which is repeated $L+1$ times within the matrix column by column. Each copy is also rotated by a specific column vector $\mathbf{w}^{\ell}$ and further shaped by a scalar $\sigma_{\ell}$ for some cases. Hence if $P=N_{t}(L+1)$, the matrix $\boldsymbol{\Omega}$ is a square matrix. The goal of the design is simplified into optimizing $\boldsymbol{\Omega}$ in (14) so that $\boldsymbol{\Omega}$ should be full rank (full spatial and frequency diversity) and $\|\operatorname{det}(\Omega)\|$ needs to be maximized. If $N_{t}<P<N_{t}(L+1)$, the goal of design is to optimize $\boldsymbol{\Omega}$ in (16) so that $\boldsymbol{\Omega} \boldsymbol{\Omega}^{\dagger}$ has full rank of $P$ (full spatial diversity but partial frequency diversity) and $\left\|\operatorname{det}\left(\boldsymbol{\Omega} \boldsymbol{\Omega}^{\dagger}\right)\right\|$ needs to be maximized.

A similar expression to (11) can be found in [13]. But the Hadamard product within (11) may conceal some valuable characteristics. Hence proposed repetition and rotation patterns shown in (14) and (16) can simplify the code design process and give us an internal observation of each specific SFBC. For example, the rate one SFBC in [12] with the assumptions of $L+1=2, N_{t}=2$ and $P=4$ is simplified as optimizing the determinant of the following matrix:

$$
\boldsymbol{\Omega}=\left[\begin{array}{cccc}
w_{1}^{0} \Delta s_{1,1} & 0 & w_{1}^{1} \Delta s_{1,1} & 0 \\
0 & w_{2}^{0} \alpha^{1} \Delta s_{2,2} & 0 & w_{2}^{1} \Delta s_{2,2} \\
w_{3}^{0} \Delta s_{3,1} & 0 & w_{3}^{1} \Delta s_{3,1} & 0 \\
0 & w_{4}^{0} \alpha^{3} \Delta s_{4,2} & 0 & w_{4}^{1} \Delta s_{4,2}
\end{array}\right]
$$

where $\Delta s_{2,1}=\Delta s_{4,1}=\Delta s_{1,2}=\Delta s_{3,2}=0$ in [12]. Then $\|\operatorname{det}(\boldsymbol{\Omega})\|=\left\|1-\phi^{2}\right\|^{2}\left\|\Delta s_{1,1} \Delta s_{2,1} \Delta s_{3,2} \Delta s_{4,2}\right\|$ where $\phi=$ $e^{j 2 \pi \delta\left(\tau_{1}-\tau_{0}\right) / T_{s}}$. The proposed SFBC in [12] will lose the diversity gain for specific channel PDP or subcarrier interval $\delta$, for example, $\phi= \pm 1$ when $\delta\left(\tau_{1}-\tau_{0}\right) / T_{s}=0.5$. The problem of diversity loss of the SFBC is not paid much attention because of the relatively complex design structure involving Hadamard products. In order to overcome diversity loss, an optimization process was proposed to adjust the subcarrier interval $\delta$ in [13].

Moreover when comparing STBC and SFBC designs, the STBC could be considered as special applications of the SFBC with highly correlated subcarriers in the MIMOOFDM subsystem. Hence we have $\mathbf{w}^{0}=\mathbf{w}^{\ell}=\mathbf{w}^{L}$. Then the matrix $\boldsymbol{\Omega}$ has the maximal diversity gain $N_{t}$ (spatial diversity only). Therefore the frequency diversity of the MIMO-OFDM system is achieved by a SFBC with properly designed repetition/rotation patterns shown in equation (14) and (16).

The minimum value of $\|\operatorname{det}(\boldsymbol{\Lambda})\|$ over all possible codeword error matrices $\Delta \mathbf{C}=\widetilde{\mathbf{C}}-\mathbf{C}$, for specific constellation $\mathcal{A}$, is denoted as coding gain $\xi$ and given by:

$$
\xi=\min _{\Delta \mathbf{C}} \frac{1}{\sqrt{N_{t}}}[\operatorname{det}(\boldsymbol{\Lambda})]^{1 / 2 P N_{r}} .
$$

3.3. Structure Analysis with Limited Knowledge of PDP. The channel PDP is assumed to be perfectly known by the transmit antennas in [13] for the purpose of optimization, and also in [8] for the purpose of high transmission rate. This assumption might be feasible for an indoor propagation scenario with relatively slow variation of channel-second order statistics. However, it is infeasible for an outdoor propagation scenario in which there are moving surrounding scatterers with large channel orders, for example, $L+1=20$ in [16]. Moreover for a multiuser scenario, each user has its own particular channel PDP, which increases the burden of feedback significantly. Hence it is more reasonable to assume that only partial PDP, for example, a limited number of paths with dominant delay power, is known by transmit antennas through data feedback or uplink transmission. The SFBC design with limited PDP can reduce both design complexity and system complexity. Therefore it is assumed that limited knowledge of PDP, only the first largest $\sigma_{\ell}^{2}$ and corresponding delays $\tau_{\ell}$ where $\ell \in[0, \ldots, \Gamma-1]$, is known by the transmit antennas and $\Gamma<L+1$.

For simplicity $P$ is assumed to be an integer multiple of $N_{t}$ (not a prerequisite) and $P=N_{t} \Gamma$. Therefore (16) should be a starting point. The first $P$ column vectors within the matrix $\boldsymbol{\Omega}$ defined in (16) are chosen to form a new matrix $\boldsymbol{\Omega}_{1}$. The remaining $N_{t}(L+1)-P$ column vectors of $\boldsymbol{\Omega}$ form a matrix $\boldsymbol{\Omega}_{2}$. Therefore, both matrices $\boldsymbol{\Omega}_{1}$ and $\boldsymbol{\Omega}_{2}$ are subblock matrices of $\boldsymbol{\Omega}$. The column vector permutation will not change the determinant of $\boldsymbol{\Omega} \boldsymbol{\Omega}^{\dagger}$ so that $\operatorname{det}(\boldsymbol{\Omega} \boldsymbol{\Omega})=\operatorname{det}\left(\boldsymbol{\Omega}_{1} \boldsymbol{\Omega}_{1}^{\dagger}+\boldsymbol{\Omega}_{2} \boldsymbol{\Omega}_{2}^{\dagger}\right)$. Let eigenvalues $\lambda_{i}(\mathbf{A})$ of an arbitrary matrix $\mathbf{A}$ be arranged in increasing order. Since $\boldsymbol{\Omega} \boldsymbol{\Omega}^{\dagger}, \boldsymbol{\Omega}_{1} \boldsymbol{\Omega}_{1}^{\dagger}$ and $\boldsymbol{\Omega}_{2} \boldsymbol{\Omega}_{2}^{\dagger}$ are Hermitian matrices and also positive semidefinite, $\lambda_{i}\left(\boldsymbol{\Omega} \boldsymbol{\Omega}^{\dagger}\right)=\lambda_{i}\left(\boldsymbol{\Omega}_{1} \boldsymbol{\Omega}_{1}{ }^{\dagger}+\boldsymbol{\Omega}_{2} \boldsymbol{\Omega}_{2}{ }^{\dagger}\right) \geq$ $\lambda_{i}\left(\boldsymbol{\Omega}_{1} \boldsymbol{\Omega}_{1}{ }^{\dagger}\right) \geq 0$ where $i \in[1, \ldots, P]$ [33]. Therefore we have 
$\operatorname{det}\left(\boldsymbol{\Omega}^{\dagger}\right) \geq \operatorname{det}\left(\boldsymbol{\Omega}_{1} \boldsymbol{\Omega}_{1}^{\dagger}\right)=\left\|\operatorname{det}\left(\boldsymbol{\Omega}_{1}\right)\right\|^{2}$. Then the determinant of $\boldsymbol{\Omega} \boldsymbol{\Omega}^{\dagger}$ has a lowerbound which can be expressed as

$$
\left\|\operatorname{det}\left(\boldsymbol{\Omega} \boldsymbol{\Omega}^{\dagger}\right)\right\| \geq\left\|\operatorname{det}\left(\boldsymbol{\Omega}_{1}\right)\right\|^{2}=\left\{\prod_{\ell=0}^{\Gamma-1} \sigma_{\ell}^{2}\right\}^{N_{t}}\|\operatorname{det}(\boldsymbol{\Psi})\|^{2},
$$

where the matrix $\Psi$ is shown as

$$
\begin{array}{r}
\boldsymbol{\Psi}=\left[\Delta \widetilde{\mathbf{S}}_{1} \circ \mathbf{w}^{0}, \ldots, \Delta \widetilde{\mathbf{S}}_{N_{t}} \circ \mathbf{w}^{0}, \ldots,\right. \\
\left.\Delta \widetilde{\mathbf{S}}_{1} \circ \mathbf{w}^{\Gamma-1}, \ldots, \Delta \widetilde{\mathbf{S}}_{N_{t}} \circ \mathbf{w}^{\Gamma-1}\right] .
\end{array}
$$

Therefore the coding gain lowerbound $\breve{\xi}$ for specific SFBC can be expressed as

$$
\xi \geq \breve{\xi}=\frac{1}{\sqrt{N_{t}}}\|\operatorname{det}(\boldsymbol{\Psi})\|^{1 / P} \prod_{\ell=0}^{\Gamma-1} \sigma_{\ell}^{1 / \Gamma} .
$$

This shows that the design of SFBC can be converted into optimizing the matrix $\boldsymbol{\Psi}$ in (20) so as to improve the coding gain lowerbound $\breve{\xi}$ given in (22). Perfect knowledge of channel PDP may not be required (or even be infeasible), but full transmit diversity order of $P$ can be guaranteed always by optimizing the coding gain lowerbound. Generally the powers of delay paths are less important than the time delays in an SFBC design because the construction of the matrix $\Psi$ is independent to the delay power. The SFBC designs proposed in this paper are based on the coding gain lowerbound with limited knowledge of PDP.

\section{Rate One Matched Rotation Precoding}

In this section a rate one SFBC with MRP is proposed. The rate one MRP has a relatively simple structure and easy optimization process when compared to the high rate SFBC. The corresponding optimization process is also discussed.
4.1. Rate One SFBC. The construction of the rate one MRP is proposed here to optimize the coding gain lowerbound $\breve{\xi}$ in (22). Assuming that $s_{p, m}=\bar{s}_{p} \mathrm{e}^{j \phi_{p, m}}$ and $\overline{\mathbf{S}}=\left[\bar{s}_{1}, \ldots, \bar{s}_{P}\right]^{\mathrm{T}}$, we have $\Delta s_{p, m}=\Delta \bar{s}_{p} \mathrm{e}^{j \phi_{p, m}}$ and

$$
\Delta \widetilde{\mathbf{S}}^{m}=\Delta \overline{\mathbf{S}} \circ \Phi^{m},
$$

where $\Delta \overline{\mathbf{S}}=\left[\Delta \bar{s}_{1}, \ldots, \Delta \bar{s}_{P}\right]^{\mathrm{T}}, \boldsymbol{\Phi}^{m}=\left[e^{j \phi_{1, m}}, \ldots, e^{j \phi_{P, m}}\right]^{T}$, and $m \in\left[1, \ldots, N_{t}\right]$. Then the matrix $\Psi$ in (22) can be expressed as

$$
\begin{array}{r}
\boldsymbol{\Psi}=\left[\Delta \overline{\mathbf{S}} \circ \boldsymbol{\Phi}^{1} \circ \mathbf{w}^{0}, \ldots, \Delta \overline{\mathbf{S}} \circ \boldsymbol{\Phi}^{N_{t}} \circ \mathbf{w}^{0}, \ldots,\right. \\
\left.\Delta \overline{\mathbf{S}} \circ \boldsymbol{\Phi}^{1} \circ \mathbf{w}^{\Gamma-1}, \ldots, \Delta \overline{\mathbf{S}} \circ \boldsymbol{\Phi}^{N_{t}} \circ \mathbf{w}^{\Gamma-1}\right] .
\end{array}
$$

The $P \times N_{t}$ matrix $\boldsymbol{\Phi}$ is defined as $\boldsymbol{\Phi}=\left[\boldsymbol{\Phi}^{1}, \ldots, \boldsymbol{\Phi}^{N_{t}}\right]$. Hence each specific rotation angle $\phi_{p, m}$ in $\boldsymbol{\Phi}$ is assigned to the $p$ th subcarrier and the $m$ th transmit antenna. Then we have

$$
\operatorname{det}\left(\Psi \Psi^{\dagger}\right)=\operatorname{det}\left(\mathbf{V} \mathbf{V}^{\dagger}\right) \prod_{p=1}^{P}\left\|\Delta \bar{s}_{p}\right\|^{2}
$$

where the square matrix $\mathbf{V}$ and the Hermitian matrix $\mathbf{V} \mathbf{V}^{\dagger}$ are shown as follows:

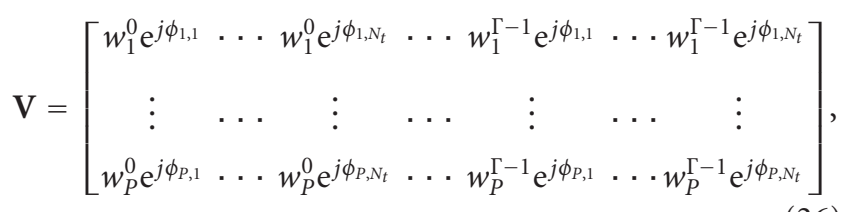

$$
\begin{aligned}
& \mathbf{V V}^{\dagger}=\left[\begin{array}{ccccc}
\Gamma & \sum_{\ell=0}^{\Gamma-1} \mathrm{e}^{-j 2 \pi \delta \tau_{\ell} / T_{s}} & \sum_{\ell=0}^{\Gamma-1} \mathrm{e}^{-j 4 \pi \delta \tau_{\ell} / T_{s}} & \cdots & \sum_{\ell=0}^{\Gamma-1} \mathrm{e}^{-j 2(P-1) \pi \delta \tau_{\ell} / T_{s}} \\
\sum_{\ell=0}^{\Gamma-1} \mathrm{e}^{-j 2 \pi \delta \tau_{\ell} / T_{s}} & \Gamma & \sum_{\ell=0}^{\Gamma-1} \mathrm{e}^{-j 2 \pi \delta \tau_{\ell} / T_{s}} & \vdots & \sum_{\ell=0}^{\Gamma-1} \mathrm{e}^{j 2 \pi(P-2) \delta \tau_{\ell} / T_{s}} \\
\vdots & \vdots & \vdots & \vdots & \vdots \\
\sum_{\ell=0}^{\Gamma-1} \mathrm{e}^{j 2 \pi(P-1) \delta \tau_{\ell} / T_{s}} & \sum_{\ell=0}^{\Gamma-1} \mathrm{e}^{j 2 \pi(P-2) \delta \tau_{\ell} / T_{s}} & \cdots & \cdots & \Gamma
\end{array}\right] \\
& \circ\left[\begin{array}{cccc}
N_{t} & \sum_{m=1}^{N_{t}} \mathrm{e}^{j\left(\phi_{1, m}-\phi_{2, m}\right)} & \cdots & \sum_{m=1}^{N_{t}} \mathrm{e}^{j\left(\phi_{1, m}-\phi_{P, m}\right)} \\
\sum_{m=1}^{N_{t}} \mathrm{e}^{j\left(\phi_{2, m}-\phi_{1, m}\right)} & N_{t} & \cdots & \sum_{m=1}^{N_{t}} \mathrm{e}^{j\left(\phi_{2, m}-\phi_{P, m}\right)} \\
\vdots & \vdots & \vdots & \vdots \\
\sum_{m=1}^{N_{t}} \mathrm{e}^{j\left(\phi_{P, m}-\phi_{1, m}\right)} & \sum_{m=1}^{N_{t}} \mathrm{e}^{j\left(\phi_{P, m}-\phi_{2, m}\right)} & \cdots & N_{t}
\end{array}\right]=\mathbf{R}_{\delta} \circ \mathbf{R}_{\phi} .
\end{aligned}
$$


The matrix $\mathbf{R}_{\delta}$ in (27) is a Hermitian Toeplitz matrix and related to time delays $\tau_{\ell}$ of dominant paths, where $\ell \in$ $[0, \ldots, \Gamma-1]$, and given subcarrier interval $\delta$. The matrix $\mathbf{R}_{\phi}=\boldsymbol{\Phi} \boldsymbol{\Phi}^{\dagger}$ is a Hermitian matrix and related to rotation angles $\phi_{p, m}$.

The principle of the MRP is to construct a proper rotation matrix $\mathbf{R}_{\phi}$ to match with matrix $\mathbf{R}_{\delta}$ so as to maximize the coding gain lowerbound. It should be pointed out that the matrix $\mathbf{R}_{\delta}$ is not a channel frequency correlation matrix, although they are similar. Thus rotation angles $\phi_{p, m}$ of $\Phi$ are determined by both time delays of propagation and subcarrier interval of subsystems. Furthermore the precoding process demonstrated in [12] can be regarded as a special application of rotation and power normalization for $\Phi$ given by

$$
\boldsymbol{\Phi}^{1}=\sqrt{2}\left[\begin{array}{llll}
1 & 0 & 1 & 0
\end{array}\right]^{T}, \quad \boldsymbol{\Phi}^{2}=\sqrt{2}\left[\begin{array}{llll}
0 & 1 & 0 & 1
\end{array}\right]^{T},
$$

and the precoding process demonstrated in [13] can also be summarized as

$$
\boldsymbol{\Phi}^{1}=\sqrt{2}\left[\begin{array}{llll}
1 & 1 & 0 & 0
\end{array}\right]^{T}, \quad \boldsymbol{\Phi}^{2}=\sqrt{2}\left[\begin{array}{llll}
0 & 0 & 1 & 1
\end{array}\right]^{T},
$$

along with the extra optimization process of subcarrier interval $\delta$ for given channel PDP.

It is also evident in (25) that the question of maximizing the coding gain lowerbound in (22) yields two independent optimization problems: $\max _{\mathcal{A}} \prod_{p=1}^{P}\left\|\Delta \bar{s}_{p}\right\|$ for specific constellation $\mathcal{A}$ and $\max _{\phi}\left\|\operatorname{det}\left(\mathbf{V} \mathbf{V}^{\dagger}\right)\right\|$ for specific correlation matrix $\mathbf{R}_{\delta}$. Hence, we denote that

$$
\begin{gathered}
\breve{\xi}_{\mathcal{A}}=\max _{\mathcal{A}} \prod_{P=1}^{P}\left\|\Delta \bar{s}_{P}\right\|^{1 / P}, \\
\breve{\xi}_{\mathrm{ECG}}=\frac{1}{\sqrt{N_{t}}}\|\operatorname{det}(\mathbf{V})\|^{1 / P} \prod_{\ell=0}^{\Gamma-1} \sigma_{\ell}^{1 / \Gamma},
\end{gathered}
$$

which is also called as extrinsic coding gain (ECG) in [13], and is always less than one. Therefore the coding gain lowerbound can be expressed as

$$
\breve{\xi}=\breve{\xi}_{\mathcal{A}} \breve{\xi}_{\mathrm{ECG}} .
$$

To maximize $\breve{\xi}_{\mathcal{A}}$ for a given constellation $\mathcal{A}$, a linear dispersion constellation code is proposed for flat fading channels [9] and adopted by some SFBCs [12, 13, 17]. The codeword $\mathbf{C}$ is precoded by a complex unitary square matrix $\Theta$ so that

$$
\overline{\mathbf{S}}=\mathbf{C} \Theta,
$$

where the codeword $\mathbf{C}=\left[c_{1}, \ldots, c_{P}\right]$ is a $1 \times P$ vector. And $c_{1}, \ldots, c_{P}$ are complex scalars chosen from a particular r-PSK or r-QAM constellation $\mathcal{A}$. It is assumed that both the real parts and the imaginary parts of $c_{1}, \ldots, c_{P}$ have a variance of $1 / 2$ and are uncorrelated, so we have $\mathbb{E}\left[c_{i} c_{i}^{*}\right]=1$ and $\mathbb{E}\left[c_{i}^{2}\right]=$ 0 where, $i \in[1, \ldots, P]$.
We will not discuss construction details of $\Theta$ here. The matrix $\Theta$ is assumed to be a Vandermonde matrix and is given by

$$
\boldsymbol{\Theta}=\frac{1}{\sqrt{P}}\left[\begin{array}{ccccc}
1 & \cdots & 1 & \cdots & 1 \\
\theta_{1} & \cdots & \theta_{i} & \cdots & \theta_{P} \\
\vdots & \vdots & \vdots & \vdots & \vdots \\
\theta_{1}^{P-1} & \cdots & \theta_{i}^{P-1} & \cdots & \theta_{P}^{P-1}
\end{array}\right],
$$

where for a QAM constellation and $P=2^{t}(t \geq 1)$, the parameters $\theta_{i}$ are given by $\theta_{i}=e^{j((4 i-3) / 2 P) \pi}$ where $i \in$ $[1, \ldots, P]$. Moreover, if $P=2^{t} 3^{q}(t \geq 1, q \geq 1)$, the parameters $\theta_{i}$ are given by $\theta_{i}=e^{j((6 i-5) / 3 P) \pi}$. Therefore we have $\breve{\xi}_{\mathcal{A}}=\Delta_{\min } / \beta$ where $\Delta_{\min }$ is the minimum Euclidean distance in constellation $\mathcal{A}$ and $\beta^{2}=P$ if $P$ is an Euler number or a power of two; otherwise $\beta^{2}=1 /\left(2^{1 / P}-1\right)$.

4.2. Optimization Process. The optimization process of the rate one MRP will focus on $\breve{\xi}_{\mathrm{ECG}}$ given by (31). Therefore a proper rotation matrix $\Phi$ is designed to maximize the coding gain lowerbound $\breve{\xi}$ for a given correlation matrix $\mathbf{R}_{\delta}$. In contrast, the optimization in [13] can be regarded as an optimization process of matrix $\mathbf{R}_{\delta}$ by adjusting the value of $\delta$ but fixing rotation matrix $\boldsymbol{\Phi}$. Adjusting the subcarrier interval $\delta$ is an efficient way of improving the subsystem performance. However, it also raises a difficulty of subcarrier grouping which must balance the averaged performance of all subsystems and the optimal performance of individual subsystem because of the conflict of subcarrier allocation.

The construction method of rotation angles $\phi_{p, m}$ might not be unique, but here for simplicity we assume that $\phi_{2,1}=0$ and $\phi_{p, m}=(p-1) \phi_{2, m}$ for $\forall p, m$. Therefore, the determinant of $\mathbf{V V}^{\dagger}$ is a function with $N_{t}-1$ variables $\phi_{1, m}$ where $m \in$ $\left[2, \ldots, N_{t}\right]$. Therefore, the coding gain lowerbound for the proposed rate one MRP is given as

$$
\begin{aligned}
\breve{\xi}=\breve{\xi}_{\mathcal{A}} \breve{\xi}_{\mathrm{ECG}}=\frac{\Delta_{\min }}{\beta \sqrt{N_{t}}} \prod_{\ell=0}^{\Gamma-1} \sigma_{\ell}^{1 / \Gamma} \\
\quad \times \prod_{\ell>l^{\prime}\left(m>m^{\prime}\right)}\left\|2 \sin \left(\frac{\pi \delta \tau_{\ell}}{T_{S}}-\frac{\pi \delta \tau_{\ell^{\prime}}}{T_{s}}+\frac{\phi_{1, m}-\phi_{1, m^{\prime}}}{2}\right)\right\|^{1 / P} \\
\leq \frac{\sqrt{\Gamma} \Delta_{\min }}{\beta} \prod_{\ell=0}^{\Gamma-1} \sigma_{\ell}^{1 / \Gamma} \leq \frac{\Delta_{\min }}{\beta},
\end{aligned}
$$

where $\ell, \ell^{\prime}, m, m^{\prime}$ are integrals, $\ell, \ell^{\prime} \in[0, \ldots, \Gamma-1]$, and $m, m^{\prime} \in\left[1, \ldots, N_{t}\right]$. The first upperbound of (35) can be achieved only with certain conditions and specific channel PDP. For instance, if $P=N_{t} \Gamma=10$, propagation delays must be uniform and given by $\tau_{\ell}=\left(3 \ell T_{s}\right) /(P \delta)$. Then rotation angles given by $\phi_{2, m}=6(L+1)(m-1) \pi / P$ can achieve this upperbound. Moreover the second upperbound (35) can be achieved with a further condition of uniform delay power so that $\sigma_{\ell}^{2}=1 / \Gamma$ for all $\ell \in[0, \ldots, \Gamma-1]$. 
As an example, the case of $P=4$ and $N_{t}=2$ is considered. A limited number of suboptimal rotation angles $\phi_{2,2}$ can be derived by differentiation of (35) and are given by

$$
\phi_{2,2}=\left\{\begin{array}{l}
k \pi \\
2 \arccos \sqrt{\frac{1}{2}+\frac{1}{2} \cos ^{2}\left(\frac{\pi \delta}{T_{s}}\left(\tau_{0}-\tau_{1}\right)\right)}+k \pi,
\end{array}\right.
$$

where $k \in \mathbb{Z}$. Then the optimal rotation angle $\phi_{2,2}$ can be obtained by comparing the coding gain lowerbound using these derived candidates.

For the case that $P$ is not an integer multiple of $N_{t}$ and $P<N_{t} \Gamma$, the process of optimization is not much different. The matrix $\boldsymbol{\Omega}_{1}$ in (20) is constructed by truncating first $P$ column vectors from the matrix $\boldsymbol{\Omega}$ and then yields the coding gain lowerbound $\breve{\xi}$. Therefore the matrix $\mathbf{V}$ will be similar to (26), but the coding gain lowerbound $\breve{\xi}$ given by (35) will be slightly different. For example, if $P=3$ and $N_{t}=2$, the targeted matrix $\mathbf{V}$ in the optimization process for the rate one MRP is given by

$$
\mathbf{V}=\left[\begin{array}{ccc}
w_{1}^{0} e^{j \phi_{1,1}} & w_{1}^{0} e^{j \phi_{1,2}} & w_{1}^{1} e^{j \phi_{1,1}} \\
w_{2}^{0} e^{j \phi_{2,1}} & w_{2}^{0} e^{j \phi_{2,2}} & w_{2}^{1} e^{j \phi_{2,1}} \\
w_{3}^{0} e^{j \phi_{3,1}} & w_{3}^{0} e^{j \phi_{3,2}} & w_{3}^{1} e^{j \phi_{3,1}}
\end{array}\right]
$$

The corresponding optimal rotation angle $\phi_{2,2}$ is given by

$$
\phi_{2,2}=k \pi-\frac{\pi \delta}{T_{s}}\left(\tau_{0}-\tau_{1}\right),
$$

where $k \in \mathbb{Z}$.

4.3. Optimization Visualization. The optimization process for the rate one MRP can be visualized by diagrams. It would be interesting to observe the optimization process for the case of $P=4$ and $N_{t}=2$ through Figure 2(a) which describes two delay paths as two points in the unit circle located in the first quadrant. Each point represents one dominant delay path. After being rotated by a certain angle $\phi_{2,2}$ clockwise, two points are then moved into the second quadrant. Hence the optimization process is to look for a best rotation angle $\phi_{2,2}$ that can maximize the product of lengths of the four dashed lines connecting these four points in Figure 2(a). Through the visualization of optimization process, it is feasible to get optimal rotation angles instinctively for some cases without complicated calculation. For example, it is easy to obtain the optimal rotation angle $\phi_{2,2}=\pi$ through Figure 2(a) and another optimal rotation angle $\phi_{2,2}=\pi / 2$ through Figure 2(b).

The visualization of optimization contains two simple steps. The first step is to put $\Gamma$ points in the unit circle whose angles, $2 \pi \delta \tau_{\ell} / T_{s}$ where $\ell \in[0, \ldots, \Gamma-1]$, are determined by corresponding time delays and subcarrier interval. The second step is to rotate these points simultaneously with a same rotation angle $\phi_{2, m}$ where $m \in\left[1, \ldots, N_{t}\right]$. And such rotations are repeated $N_{t}$ times and each time creates a new set of $\Gamma$ points. Therefore after these rotations, a total of $N_{t}$ sets corresponding to $N_{t} \Gamma$ points are created and

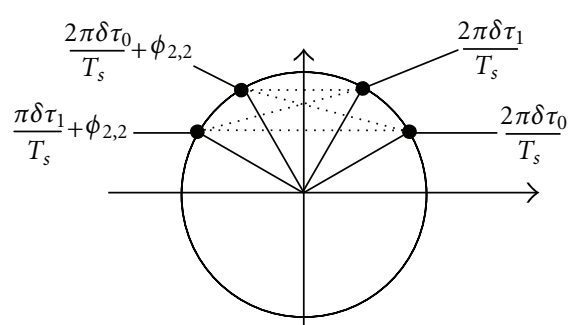

(a)

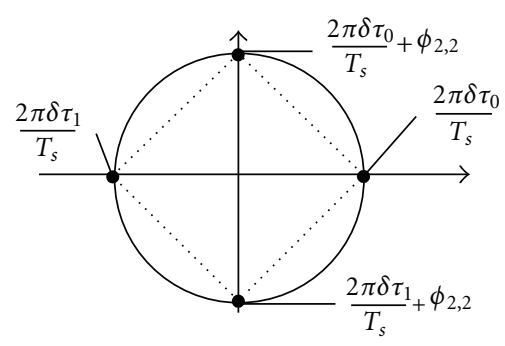

(b)

Figure 2: Visualization of optimization for the case $P=4$ and $N_{t}=$ 2.

spread around the unit circle. Therefore there are $\Gamma^{2} N_{t}\left(N_{t}-\right.$ 1)/2 lines connecting these points among different sets, for example, four lines in Figure 2. Beware that the connection lines between points within a same set are irrelevant to the optimization process because these lines are unchangeable (determined by the time delays of channel). The angle $\phi_{2,1}$ is assumed to be zero here so that only $N_{t}-1$ rotations are optimized.

The optimization process is to maximize the product of lengths of these connection lines. The optimal case is that total $N_{t} \Gamma$ points are uniformly distributed around the unit circle with an exact separation angle $2 \pi /\left(N_{t} \Gamma\right)$. This case gives the best performance for the specific subsystem and achieves the coding gain upperbound derived in (35) and [12]. Moreover, the STBC proposed in [34] has some similarity with the rate one MRP in terms of optimization strategy. The optimal constellation rotation in [34] is designed for a particular constellation with a single rotation and space diversity, but the rate one MRP is designed for particular propagation channel (independent of constellation) with multiple rotations and space-frequency diversity. Hence the rate one MRP can be visualized as a SFBC optimizing "channel Euclidean distance."

4.4. Examples. As an example we determine optimal rotation angles for a multipath fading model, COST207 six-ray power delay profile for typical urban scenario [27] described in Table 1. The power of delays of COST207 is sorted in a decreasing order. The MIMO-OFDM system has two transmit antennas, 512 subcarriers and a bandwidth of $16 \mathrm{MHz}$. The subcarrier interval $\delta$ in the MIMO-OFDM subsystem is assumed to be $\delta=\lfloor 512 / P\rfloor$. Then the MRP has only one unknown variable $\phi_{2,2}$, and $\phi_{p, 2}=(p-1) \phi_{2,2}$ 
TABLE 1: COST207 typical urban six-ray power delay profile.

\begin{tabular}{lcccccc}
\hline Time delay $(\mu s)$ & 0.2 & 0.5 & 0 & 1.6 & 2.3 & 5.0 \\
\hline Delay power & 0.379 & 0.239 & 0.189 & 0.095 & 0.061 & 0.037 \\
\hline
\end{tabular}

TABLe 2: Optimal rotation angle for COST207.

\begin{tabular}{ccc}
\hline$P$ & $\phi_{2,2}$ & $\breve{\xi}_{\text {ECG }}$ \\
\hline 3 & $107 \pi / 180$ & 0.6865 \\
4 & $\pi$ & 0.7566 \\
5 & $129 \pi / 180$ & 0.5228 \\
6 & $141 \pi / 180$ & 0.7082 \\
\hline
\end{tabular}

for all $p \in[1, \ldots, P]$. It is assumed that only limited PDP of COST207 MIMO channel, that is, time delay $\tau_{\ell}$ shown in Table 1 where $\ell \in[0, \ldots, \Gamma-1]$, is actually known by the transmit antennas. It is also assumed that $\Gamma=\left\lceil P / N_{t}\right\rceil$ where $\lceil a\rceil$ denotes the smallest integer greater than or equal to $a$. Hence if $P=3,4$, then $\Gamma=2$ delays are known by the transmit antennas. And if $P=5,6$ then $\Gamma=$ 3.

Since the proposed rate one MRP is composed of two independent optimization processes and $\breve{\xi}_{\mathcal{A}}$ is only related to the constellation $\mathcal{A}$, we focus on $\breve{\xi}_{\text {ECG }}$ only which is highly related to the specific channel PDP known by the transmit antennas. Figure 3 shows the variations of $\breve{\xi}_{\mathrm{ECG}}$ of the MRP for a variety of values of $\phi_{2,2}$ and $P$. All peak points in Figure 3 with corresponding coordinates of $\phi_{2,2}$ and $\breve{\xi}_{\text {ECG }}$ are summarized in Table 2. The optimization of coding gain lowerbound $\breve{\xi}$ can be used to search for an approaching optimal performance since only partial PDP is known. But full transmit diversity can always be guaranteed. Moreover, full transmit diversity is achieved for same cases even if the coding gain lowerbound $\breve{\xi}$ equals to zero. Hence the condition that the lowerbound $\breve{\xi}$ should be greater than zero is a sufficient condition to achieve full transmit diversity. The optimal rotation angle $\phi_{2,2}$ is varied from case to case. At last the selection of column vectors for $\boldsymbol{\Omega}_{1}$ will affect the design process and results of optimization. But it is known that if more column vectors are built inside $\boldsymbol{\Omega}_{1}$ (it also means better knowledge of PDP at the transmit end), the optimization process will be closer to optimal.

On the other hand the optimization process of subcarrier interval $\delta$ is still feasible for the proposed rate one MRP. Figure 4 shows the changes of the $\breve{\xi}_{\mathrm{ECG}}$ of the rate one MRP for a variety of values of $\phi_{2,2}$ and $\delta$. For arbitrary subcarrier interval $\delta$, the rotation angle $\phi_{2,2}$ can be adjusted to achieve the optimal performance. Subcarrier interval $\delta$ is fixed to $\left\lfloor N_{c} / P\right\rfloor$ in this paper considering limited choices of subcarrier interval $\delta$ because of the conflict of subcarrier allocation if the performance of all users in a multiuser scenario needs to be optimized simultaneously by adjusting subcarrier interval.

Remark 2. The rate one MRP with limited PDP is proposed for the circumstance that the transmit antennas have

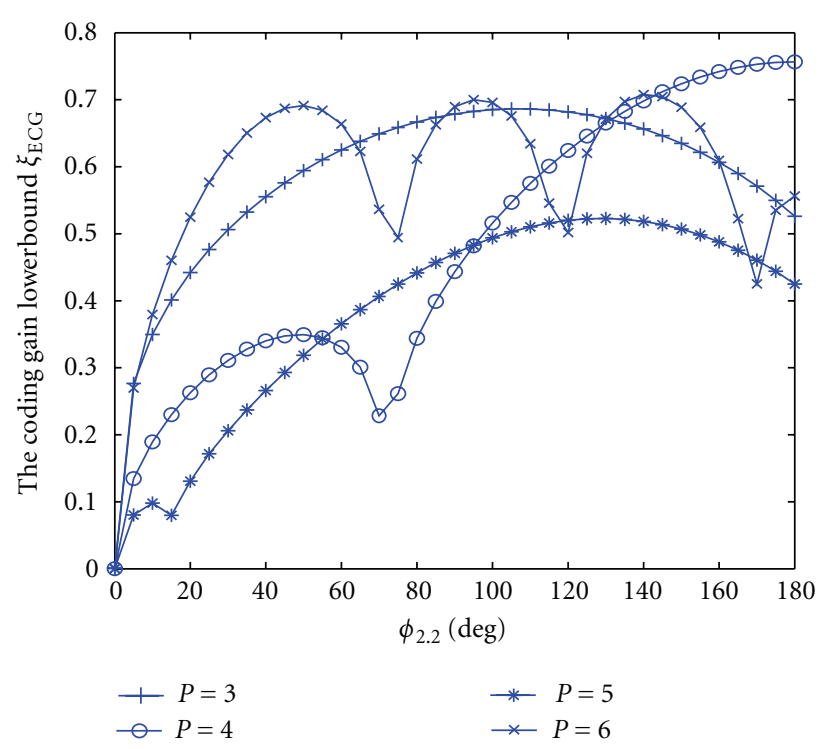

FIGURE 3: $\breve{\xi}_{\text {ECG }}$ of rate one MRP versus rotation angle $\phi_{2,2}$ for a MIMO-OFDM system with $\delta=\lfloor 512 / P\rfloor, N_{t}=2, N_{c}=512$, and given COST207 typical urban six-ray power delay profile.

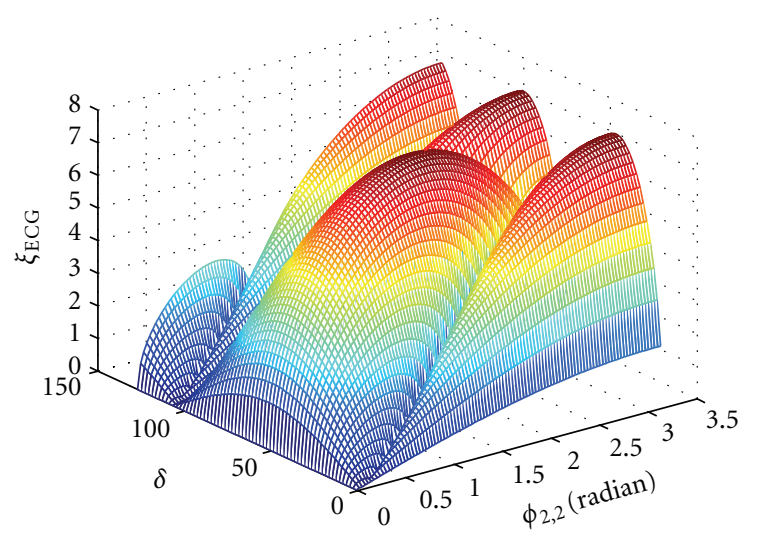

FIGURE 4: $\breve{\xi}_{\text {ECG }}$ of the rate one MRP versus rotation angle $\phi_{2,2}$ and $\delta$ for a MIMO-OFDM system with $P=4, N_{t}=2, N_{c}=512, \Gamma=$ $P / N_{t}=2$ and given COST207 typical urban six-ray power delay profile.

only partial or the imperfect knowledge of the channel PDP through the feedback from the receive antennas or uplink transmission. It is capable of reducing both system complexity and SFBC design complexity significantly. Better optimization process requires more knowledge of channel PDP. Moreover, the rate one MRP can overcome the drawback of diversity loss in [12] for specific propagation scenarios, and mitigate the limitations of subcarrier interval and subcarrier grouping. It can always achieve full transmit diversity and approach to optimal performance.

\section{Multirate Matched Rotation Precoding}

In this section, the multirate SFBC with MRP is proposed. It has better spectral efficiency when compared to the rate one 
MRP, and better performance if the same bit transmission rate is assumed. It also can achieve relatively smooth balance between the performance and the transmission rate without a significant configuration change. The optimization process of the proposed multirate MRP is also discussed.

5.1. Multirate SFBC. The multirate MRP is proposed here to optimize the coding gain lowerbound $\breve{\xi}$ denoted in (22). Assuming that $s_{p, m}=\bar{s}_{p, m} e^{j \phi_{p, m}}$ and $\overline{\mathbf{S}}^{m}=\left[\bar{s}_{1, m}, \ldots, \overline{\boldsymbol{s}}_{P, m}\right]^{T}$, we have $\Delta s_{p, m}=\Delta \bar{s}_{p, m} e^{j \phi_{p, m}}$ and

$$
\Delta \widetilde{\mathbf{S}}_{m}=\Delta \overline{\mathbf{S}}^{m} \circ \boldsymbol{\Phi}^{m}
$$

where $\Delta \overline{\mathbf{S}}^{m}=\left[\Delta \bar{s}_{1, m}, \ldots, \Delta \bar{s}_{P, m}\right]^{T}, \boldsymbol{\Phi}^{m}=\left[e^{j \phi_{1, m}}, \ldots, e^{j \phi_{P, m}}\right]^{T}$ and $m \in\left[1, \ldots, N_{t}\right]$. The matrix $\Psi$ in (22) can be expressed as

$$
\begin{aligned}
\boldsymbol{\Psi}= & {\left[\Delta \overline{\mathbf{S}}^{1} \circ \boldsymbol{\Phi}^{1} \circ \mathbf{w}^{0}, \ldots, \Delta \overline{\mathbf{S}}^{N_{t}} \circ \boldsymbol{\Phi}^{N_{t}} \circ \mathbf{w}^{0}, \ldots,\right.} \\
& \left.\Delta \overline{\mathbf{S}}^{1} \circ \boldsymbol{\Phi}^{1} \circ \mathbf{w}^{\Gamma-1}, \ldots, \Delta \overline{\mathbf{S}}^{N_{t}} \circ \boldsymbol{\Phi}^{N_{t}} \circ \mathbf{w}^{\Gamma-1}\right] .
\end{aligned}
$$

Then $\Psi \Psi^{\dagger}$ is shown in (41) as follows:

$$
\begin{aligned}
& \Psi \Psi^{\dagger}=\left[\begin{array}{ccccc}
\Gamma & \sum_{\ell=0}^{\Gamma-1} \mathrm{e}^{-j 2 \pi \delta \tau_{\ell} / T_{s}} & \sum_{\ell=0}^{\Gamma-1} \mathrm{e}^{-j 4 \pi \delta \tau_{\ell} / T_{s}} & \cdots & \sum_{\ell=0}^{\Gamma-1} \mathrm{e}^{-j 2(P-1) \pi \delta \tau_{\ell} / T_{s}} \\
\sum_{\ell=0}^{\Gamma-1} \mathrm{e}^{j 2 \pi \delta \tau_{\ell} / T_{s}} & \Gamma & \sum_{\ell=0}^{\Gamma-1} \sigma_{\ell}^{2} \mathrm{e}^{-j 2 \pi \delta \tau_{\ell} / T_{s}} & \cdots & \sum_{\ell=0}^{\Gamma-1} \mathrm{e}^{-j 2 \pi(P-2) \delta \tau_{\ell} / T_{s}} \\
\vdots & \vdots & \vdots & \vdots & \vdots \\
\sum_{\ell=0}^{\Gamma-1} \mathrm{e}^{\mathrm{j} 2(P-1) \pi \delta \tau_{\ell} / T_{s}} & \sum_{\ell=0}^{\Gamma-1} \mathrm{e}^{j 2 \pi(P-2) \delta \tau_{\ell} / T_{s}} & \cdots & \cdots & \Gamma
\end{array}\right] \\
& \circ\left[\begin{array}{cccc}
\sum_{m=1}^{N_{t}}\left\|\Delta \bar{s}_{1, m}\right\|^{2} & \sum_{m=1}^{N_{t}}\left(\Delta \bar{s}_{1, m} \Delta \bar{s}_{2, m}^{*} \mathrm{e}^{j\left(\phi_{1, m}-\phi_{2, m}\right)}\right) & \cdots & \sum_{m=1}^{N_{t}}\left(\Delta \bar{s}_{1, m} \Delta \bar{s}_{P, m}^{*} \mathrm{e}^{j\left(\phi_{1, m}-\phi_{P, m}\right)}\right) \\
\sum_{m=1}^{N_{t}}\left(\Delta \bar{s}_{2, m} \Delta \bar{s}_{1, m}^{*} \mathrm{e}^{j\left(\phi_{2, m}-\phi_{1, m}\right)}\right) & \sum_{m=1}^{N_{t}}\left\|\Delta \bar{s}_{2, m}\right\|^{2} & \cdots & \sum_{m=1}^{N_{t}}\left(\Delta \bar{s}_{2, m} \Delta \bar{s}_{P, m}^{*} \mathrm{e}^{j\left(\phi_{2, m}-\phi_{P, m}\right)}\right) \\
\vdots & \vdots & \vdots & \vdots \\
\sum_{m=1}^{N_{t}}\left(\Delta \bar{s}_{1, m} \Delta \bar{s}_{P, m}^{*} \mathrm{e}^{j\left(\phi_{P, m}-\phi_{1, m}\right)}\right) & \sum_{m=1}^{N_{t}}\left(\Delta \bar{s}_{P, m} \Delta \bar{s}_{2, m}^{*} \mathrm{e}^{j\left(\phi_{P, m}-\phi_{2, m}\right)}\right) & \cdots & \sum_{m=1}^{N_{t}}\left\|\Delta \bar{s}_{P, m}\right\|^{2}
\end{array}\right] \\
& =\mathrm{R}_{\delta} \circ \mathrm{R}_{\psi} \text {. }
\end{aligned}
$$

The rotation matrix $\Phi_{R}$ for the symbol transmission rate $R$ is denoted as $\boldsymbol{\Phi}_{R}=\left[\boldsymbol{\Phi}^{1}, \ldots, \boldsymbol{\Phi}^{N_{t}}\right]$.

The Hermitian matrix $\boldsymbol{\Psi} \Psi^{\dagger}$ is the Hadamard product of two matrices $\mathbf{R}_{\delta}$ and $\mathbf{R}_{\psi}$ denoted in (41). The matrix $\mathbf{R}_{\delta}$ is related to both time delays $\tau_{\ell}$ of paths and subcarrier interval $\delta$. But the matrix $\mathbf{R}_{\psi}$ of the multirate MRP is more complicated than the matrix $\mathbf{R}_{\phi}$ denoted in (27). It is related to the proposed rotation matrix $\Phi_{R}$ and also the specific constellation $\mathcal{A}$.

Supposed that the vector $\overline{\mathbf{S}}$ is defined as $\overline{\mathrm{S}}=$ $\left[\left(\overline{\mathbf{S}}^{1}\right)^{T}, \ldots,\left(\overline{\mathbf{S}}^{N_{t}}\right)^{T}\right]$. The precoding process of the multirate MRP with transmission rate $R$ is given by

$$
\overline{\mathbf{S}}=\mathbf{C} \Theta_{R},
$$

where the codeword $\mathbf{C}=\left[c_{1}, \ldots, c_{Q}\right]$ is a $1 \times Q$ vector where $c_{1}, \ldots, c_{Q}$ are complex scalars chosen from a particular r-PSK or r-QAM constellation $\mathcal{A}$. The symbol transmission rate is denoted as $R=Q / P$. It is assumed that both the real parts and the imaginary parts of $c_{1}, \ldots, c_{Q}$ have a variance of $1 / 2$ and are uncorrelated, so we have $\mathbb{E}\left[c_{i} c_{i}^{*}\right]=1$ and $\mathbb{E}\left[c_{i}^{2}\right]=0$ where $i \in[1, \ldots Q]$.

The matrix $\boldsymbol{\Theta}_{R}$ is an $Q \times N_{t} P$ complex coding matrix satisfying the following power normalization equation:

$$
\operatorname{trace}\left(\boldsymbol{\Theta}_{R} \boldsymbol{\Theta}_{R}^{\dagger}\right)=N_{t} P
$$

Hence the codeword $\mathrm{C}$ is dispersed from $\mathrm{Q}$ dimensional vector to $N_{t} P$ transmission data across both frequency and space domains. The value of integer $Q$ can be chosen from 1 to $N_{t} P$ so that the symbol transmission rate $R$ can be varied from $1 / P$ up to $N_{t}$.

When the MIMO-OFDM subsystem achieves the highest transmission rate $R=N_{t}$, then $Q=N_{t} P$. The matrix $\Theta_{N_{t}}$ 
is a unitary square matrix and assumed to a Vandermonde matrix given by (34). Hence we have

$$
\Theta_{N_{t}}=\Theta .
$$

When the bit error rate (BER) performance of the MIMO-OFDM subsystem is worse than the expected performance, the transmission rate $R$ can be reduced to achieve better BER performance without decreasing constellation size or significantly changing the coding structure. Thus when $R \leq N_{t}, Q=R P$ and $\boldsymbol{\Theta}_{R}$ is a $R P \times N_{t} P$ matrix. The coding matrix $\boldsymbol{\Theta}_{R}$ can be obtained by simply truncating first $R P$ row vectors from the coding matrix $\boldsymbol{\Theta}_{N_{t}}$ and applying power normalization using (43). Hence the matrix $\boldsymbol{\Theta}_{R}$ is given by

$$
\boldsymbol{\Theta}_{R}=\sqrt{\frac{N_{t}}{R}} \boldsymbol{\Theta}_{Q \times P N_{t}},
$$

where $\boldsymbol{\Theta}_{Q \times P N_{t}}$ is a truncated matrix from $\boldsymbol{\Theta}_{N_{t}}$.

Matrices $\boldsymbol{\Theta}_{R}$ and $\boldsymbol{\Phi}_{R}$ are key matrices for the multirate MRP where the rate $R$ ranges from $1 / P$ to $N_{t}$. They can even summarize coding structures of most existing SFBCs as a variety of matrix pairs $\boldsymbol{\Theta}_{R}$ and $\boldsymbol{\Phi}_{R}$. The matrix $\boldsymbol{\Theta}_{R}$ can disperse the information of a codeword $\mathrm{C}$ into $N_{t} P$ subchannels but it cannot guarantee full diversity gain or the optimal performance. The matrix $\boldsymbol{\Phi}_{R}$ can guarantee full transmit diversity and optimize the coding gain simultaneously for specific channel PDP known by transmit antennas. Hence it is strongly related to constellation $\mathcal{A}$, correlation matrix $\mathbf{R}_{\delta}$ and designed matrix $\boldsymbol{\Theta}_{R}$.

Remark 3. The rotation matrix $\Phi_{\mathrm{R}}$ for arbitrary rate $R$ can be assumed to be the same as the matrix $\Phi_{\mathrm{N}_{\mathrm{t}}}$ designed for the highest rate $N_{t}$, that is, $\boldsymbol{\Phi}_{R}=\boldsymbol{\Phi}_{N_{t}}$ for all $R$. Therefore if matrices $\boldsymbol{\Theta}_{N_{t}}$ and $\boldsymbol{\Phi}_{N_{t}}$ can achieve full transmit diversity $P$ at the highest rate $N_{t}$ for the MIMO-OFDM subsystem; full transmit diversity can be guaranteed at each transmission rate $\mathrm{R}$ if the matrices $\boldsymbol{\Theta}_{R}$ are derived from $\boldsymbol{\Theta}_{N_{t}}$ and $\boldsymbol{\Phi}_{R}=$ $\boldsymbol{\Phi}_{N_{t}}$. Hence the multirate MRP can generate a series of lower rate SFBCs and reduce design complexity significantly. The explanation is the following.

The codeword error $\Delta \mathbf{C}$ for the transmission rate $R$ can be obtained by assigning zeros to the last $\left(N_{t}-R\right) P$ symbols of $\Delta \mathbf{C}$ for the transmission rate $N_{t}$. Thus the set of $\Delta \mathbf{C}$ for the rate $R$ actually becomes a subset of $\Delta \mathbf{C}$ for the rate $N_{t}$. Therefore, for the lower transmission rate $R$, the size of subset of $\Delta \mathrm{C}$ is smaller giving a larger coding gain and better BER performance. The rotation matrix $\boldsymbol{\Phi}_{R}$ can be either specially designed for a specific rate $R$ and symbol constellation $\mathcal{A}$, or kept unchanged as $\boldsymbol{\Phi}_{N_{t}}$ for simplicity. The matrix $\boldsymbol{\Theta}_{R}$ can be obtained by simply truncation and power normalization from the matrix $\boldsymbol{\Theta}_{N_{t}}$. Full transmit diversity of $P$ is always guaranteed in the multirate MRP.

5.2. Optimization Process. The optimization process of the multirate MRP will be more complicated than the rate one MRP. The determinant of $\Psi^{\dagger} \Psi^{\dagger}$ given by (41) is affected by elements of both matrix $\mathbf{R}_{\delta}$ and matrix $\mathbf{R}_{\psi}$. The subcarrier interval is fixed to $\delta=\left\lfloor N_{c} / P\right\rfloor$ so that the matrix $\mathbf{R}_{\delta}$ is unchanged. The matrix $\mathbf{R}_{\psi}$ is related with specific constellation $\mathcal{A}$, designed matrix $\boldsymbol{\Theta}_{R}$, and rotation matrix $\boldsymbol{\Phi}_{R}$. The optimization process adjusts both matrices $\boldsymbol{\Theta}_{R}$ and $\Phi_{R}$ simultaneously.

The matrix $\boldsymbol{\Theta}_{R}$ is given by (44) and (45) according to the transmission rate $R$. And for the simplicity, we assume that $\phi_{p, m}=(p-1)(m-1) \phi_{2,2}$ for all $p, m$ in $\boldsymbol{\Phi}_{R}$. The determinant of $\Psi \Psi^{\dagger}$ given by (41) is a polynomial equation with only one variable $\phi_{2,2}$ and $\operatorname{det}\left(\Psi \Psi^{\dagger}\right)=\|\operatorname{det}(\boldsymbol{\Psi})\|^{2}$. The optimization process of the multirate MRP has only one unknown variable $\phi_{2,2}$ for an arbitrary MIMO-OFDM subsystem.

Remark 4. If $\mathrm{e}^{j \phi_{2,2}}$ is an algebraic number of degree greater than $N_{t} P^{2}$ over $\mathcal{K}$ which is the extension field containing all the entries of $\Theta_{R}$, the ring of complex integers $\mathbb{Z}(j)$, and $\mathrm{e}^{j 2 \pi \delta \tau_{\ell} / \mathrm{T}_{\mathrm{s}}}$ where $\ell \in[0, \ldots, \Gamma-1]$, then full transmit diversity $P$ is guaranteed for all QAM constellation. This property can be derived from the determinant expression of $\Psi$ denoted in (41).

The high rate SFBC proposed in [8] has a similar rule as to above. But the proposed multirate MRP has utilized a rotation matrix $\boldsymbol{\Phi}_{R}$ designed for the scenario with limited channel PDP, and also defined a smaller extension field $\mathcal{K}$ compared to [8]. Moreover, despite relatively high complexity and difficulty in the optimization process, the multirate MRP always takes advantage of a portion of information about propagation paths for arbitrary channel PDP, so that it is feasible to generate a decision table for optimization in advance at the transmit end. Therefore such a table can be stored and reused without the requirement of another calculation.

5.3. Examples. As an example the optimal rotation angle $\phi_{2,2}$ is generated for COST 207 typical urban scenario defined in Table 1. The propagation channel knowledge is assumed to be limited so that only $\tau_{\ell}$, where $\ell \in[0, \ldots, \Gamma-1]$, are perfectly known by the transmit antennas where $\Gamma=$ 2. The MIMO-OFDM system has two transmit antennas, 512 subcarriers, and a bandwidth $16 \mathrm{MHz}$. Each MIMOOFDM subsystem has four well-separated subcarriers and the subcarrier interval is assumed to $\delta=128$.

For a QPSK constellation, the coding gain lowerbound $\breve{\xi}$ of the multirate MRP is maximized by a computer search over $\phi_{2,2}$ varying from 0 to $\pi$. The step size is $\pi / 180$ so that the algebraic degree meets the condition of Remark 4 . The optimal rotation angles $\phi_{2,2}$ for a variety of transmission rates $R$ are shown in Table 3. Moreover the optimal rotation angle $\phi_{2,2}=168 \pi / 180$ gives the largest coding gain lowerbound at the highest transmission rate $R=2$. Hence the rotation angle $\phi_{2,2}$ can be fixed to $168 \pi / 180$ without jeopardizing the transmission diversity according to Remark 3 . Therefore the coding gain lowerbound $\breve{\xi}$ corresponding to $\phi_{2,2}=$ $168 \pi / 180$ for different rates $R$ are also included in Table 3 for comparison.

It is also feasible to yield a decision table for the multirate MRP in advance since only a limited number of propagation paths are needed for the optimization process. Figure 5 is 
TABLE 3: Transmission rate $R$ versus optimal rotation angle $\phi_{2,2}$ and corresponding coding gain lowerbound $\breve{\xi}$ for the Multirate MRP in a MIMO-OFDM subsystem with $P=4, N_{t}=\Gamma=2$, and QPSK for COST 207 Typical urban six-ray power delay profile.

\begin{tabular}{lccc}
\hline$R$ & $\phi_{2,2}$ & $\breve{\xi}$ & $\breve{\xi}$ when $\phi_{2,2}=168 \pi / 180$ \\
\hline $1 / 4$ & $\pi$ & 1.07 & 1.0626 \\
$2 / 4$ & $\pi$ & 0.7566 & 0.7514 \\
$3 / 4$ & $164 \pi / 180$ & 0.5660 & 0.3493 \\
$4 / 4$ & $154 \pi / 180$ & 0.4231 & 0.3025 \\
$5 / 4$ & $95 \pi / 180$ & 0.3048 & 0.2706 \\
$6 / 4$ & $99 \pi / 180$ & 0.2641 & 0.2337 \\
$7 / 4$ & $165 \pi / 180$ & 0.2076 & 0.2004 \\
$8 / 4$ & $168 \pi / 180$ & 0.1874 & 0.1874 \\
\hline
\end{tabular}

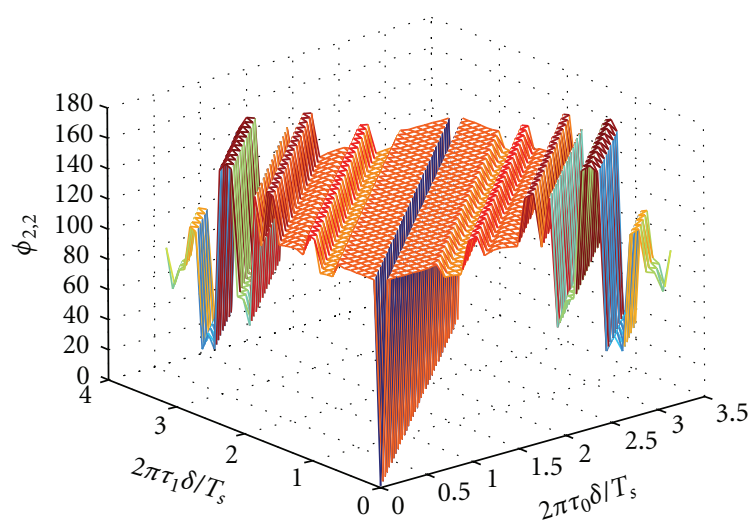

Figure 5: Decision table of the optimal rotation angle $\phi_{2,2}$ for multirate MRP in a MIMO-OFDM subsystem with $P=4, R=$ $N_{t}=\Gamma=2$, and BPSK constellation.

an example of a decision table for a BPSK constellation and the transmission rate $R=2$. The $z$-axis in the figure shows the optimal rotation angle $\phi_{2,2}$ for a specmific channel PDP which has two dominant delays marked by corresponding $x$ and $y$ coordinates. Hence it would be easy to determine the optimal rotation angle $\phi_{2,2}$ for an arbitrary MIMOOFDM subsystem and channel PDP once such a decision table has been generated. It is also shown that if $2 \pi \tau_{0} \delta / T_{s}=$ $2 \pi \tau_{1} \delta / T_{s}+2 k \pi$ where $k \in \mathbb{Z}$, the coding gain lowerbound $\breve{\xi}$ would be equal to zero with a potential loss of diversity gain.

\section{Simulation Results}

To illustrate the performance of the rate one MRP and the multirate MRP, we performed some simulations and made comparisons with existing SFBC given in [12]. For example, if $P=3$, transmitted symbols $\hat{\mathbf{S}}\left(P \times N_{t}\right.$ matrices $)$ using the rate one MRP and the SFBC in [12] are given by the following, respectively,

$$
\widehat{\boldsymbol{S}}=\left[\begin{array}{cc}
\bar{s}_{1} & \bar{s}_{1} \\
\bar{s}_{2} & \bar{s}_{2} \mathrm{e}^{j \phi_{2,2}} \\
\bar{s}_{3} & \bar{s}_{3} \mathrm{e}^{2 j \phi_{2,2}}
\end{array}\right], \quad \widehat{\mathbf{S}}=\left[\begin{array}{cc}
\sqrt{2} \bar{s}_{1} & 0 \\
0 & \sqrt{2} \bar{s}_{2} \\
\sqrt{2} \bar{s}_{3} & 0
\end{array}\right],
$$

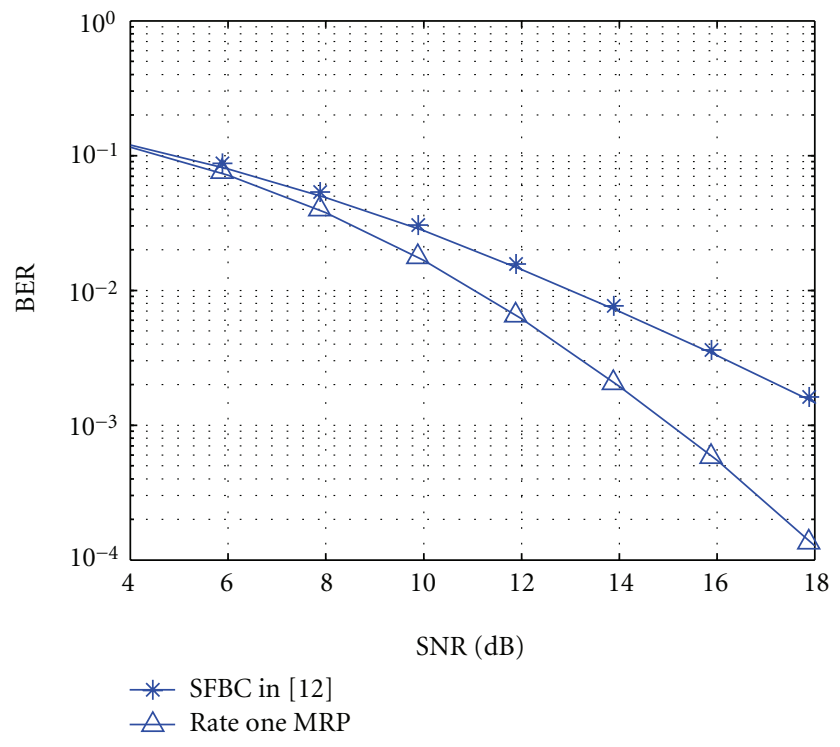

FIGURE 6: Performance of the rate one MRP and the SFBC in [12] for the MIMO-OFDM system with $N_{t}=2, N_{r}=1, N_{c}=512$, and $\delta=128$ in the propagation scenario with uniform PDP of two paths.

where $\overline{\mathbf{S}}=\left[\bar{s}_{1}, \bar{s}_{2}, \bar{s}_{3}\right]=\mathbf{C} \Theta$. The constellation $\mathcal{A}$ of the codeword $\mathbf{C}$ is chosen to be quaternary phase-shift keying (QPSK) or 16QAM. The precoding matrix $\Theta$ is given by (34). The optimal rotation angles $\phi_{2,2}$ of the rate one MRP are given by the second column of Table 2. The channel state information $\widetilde{\mathbf{H}}$ and the rotation angles $\phi_{2,2}$ are perfectly known by the receive antennas. However limited knowledge of channel PDP is available at the transmit side. The decoding process is unified at first in all simulations according to [35]. The same sphere decoding $[36,37]$ is used at the receive antennas for each subsystem and each SFBC. The bit error rate (BER) performance is averaged over all MIMO-OFDM subsystems and channel realizations.

6.1. Diversity Loss. The SFBC without optimization process might lose the diversity gain for specific propagation scenario. It has not been completely recognized but this problem can be overcome by adjusting subcarrier interval $\delta$ or applying proposed MRP. We assume that the MIMOOFDM system has $N_{t}=2, N_{r}=1,16 \mathrm{MHz}$ Bandwidth, and $N_{c}=512$ subcarriers. Each subsystem has $P=4$ wellseparated subcarriers and a fixed subcarrier interval $\delta=128$. The propagation scenario has only two delay paths or two dominant delay paths. It is shown in (14) that for this kind of system setting and propagation scenario, the powers of paths are irrelevant to the SFBC design process. Hence it is assumed that the wireless channel has uniform delay power and $\sigma_{\ell}^{2}=1 / 2$ for simplicity. The time delays are assumed to $\tau_{0}=0$ and $\tau_{1}=2 T_{s} / N_{c}$. The rate one MRP is compared with the SFBC in [12] with an exactly same system configuration. The optimal rotation angle $\phi_{2,2}$ of the rate one MRP for this specific propagation scenario is $\pi / 2$ which can be seen in Figure 2(b). 
Figure 6 shows clearly the improvement of the rate one MRP. The SFBC in [12] cannot achieve full transmit diversity for this specific propagation scenario but the proposed rate one MRP can provide a significant improvement. The Hadamard product in (11) might conceal the loss of frequency diversity. Hence we should be cautious about judging the performance of specific SFBC, which might be good for some cases but lose diversity gain for the others. The optimization process is important for all existing SFBCs because of potential diversity loss, through either adjusting subcarrier interval $\delta$, or applying the proposed MRP, or even both.

6.2. Rate One MRP. To show the performance of the rate one MRP with limited feedback, a more practical scenario COST207 typical urban six-ray PDP is considered and defined in Table 1. The MIMO-OFDM system has $N_{t}=2$, $N_{r}=1,16 \mathrm{MHz}$ Bandwidth, and $N_{c}=512$ subcarriers. Each subsystem has $P$ well-separated subcarriers and a fixed subcarrier interval $\delta=\lfloor 512 / P\rfloor$. The rest unallocated subcarriers $(<P)$ could be used as subcarrier guard interval. Limited knowledge of channel PDP is fed back to the transmit antennas. So it is assumed that only time delays $\tau_{\ell}$ where $\ell \in[0, \ldots, \Gamma-1]$ are known by the transmit antennas. It is also assumed that $\Gamma=\left\lceil P / N_{t}\right\rceil$.

It is shown in Figure 7 that the performance of the rate one MRP has better performance than the SFBC in [12] when $P=3$ and 6 , and the same performance when $P=$ 4 for COST207 typical urban scenario. The performance gain of the rate one MRP is roughly about $0.5 \mathrm{~dB}$ at a BER of $10^{-3}$ when $P=3$. And the performance gain is about $0.2 \mathrm{~dB}$ when $P=6$. The performances of both SFBCs are same when $P=4$ but we could observe the advantage of the rate one MRP for other channel PDP from Figure 6. The observation confirms that the rate one MRP does improve BER performance by optimizing coding gain lowerbound $\breve{\xi}$ for some cases, even when the knowledge of channel PDP is limited. Therefore the rate one MRP is capable of providing more design freedom and better system performance compared to [12, 13]. Furthermore the information required by the optimization process can be mitigated by the proposed SFBC design with limited knowledge of PDP.

6.3. Multirate MRP. The multirate SFBC following the coding matrix (39) is investigated in Figure 8 for the MIMOOFDM system with $N_{t}=2, N_{r}=2, P=4, \delta=128$, and $N_{c}=512$. The constellations of QPSK and 16QAM are applied. For example, transmitted symbols $\widehat{\mathbf{S}}\left(P \times N_{t}\right.$ matrices) using the multirate MRP are given by

$$
\widehat{\mathbf{S}}=\left[\begin{array}{cc}
\bar{s}_{1,1} & \bar{s}_{1,2} \\
\bar{s}_{2,1} & \bar{s}_{2,2} \mathrm{e}^{j \phi_{2,2}} \\
\bar{s}_{3,1} & \bar{s}_{3,2} \mathrm{e}^{2 j \phi_{2,2}} \\
\bar{s}_{4,1} & \bar{s}_{4,2} \mathrm{e}^{3 j \phi_{2,2}}
\end{array}\right],
$$

where $\overline{\mathbf{S}}$ is precoded by the matrix $\boldsymbol{\Theta}_{R}$ which is specified by (44) and (45). And the rotation angle $\phi_{2,2}$ of multirate MRP

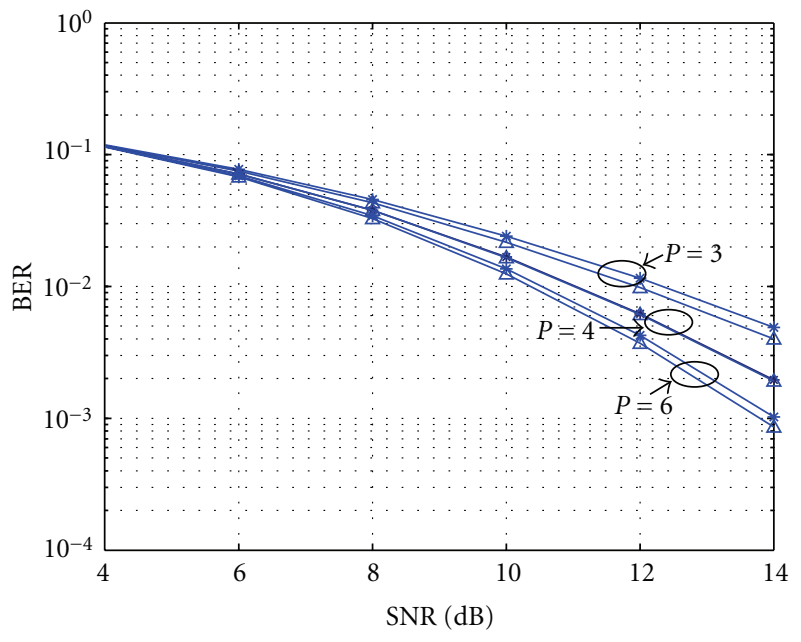

FIGURE 7: Performance of the MRP marked by $\Delta$ and the SFBC in [12] marked by $*$ for the MIMO-OFDM system with $N_{t}=2, N_{r}=$ $1, N_{c}=512$, and $\delta=\lfloor 512 / P\rfloor$ in COST207 typical urban scenario.

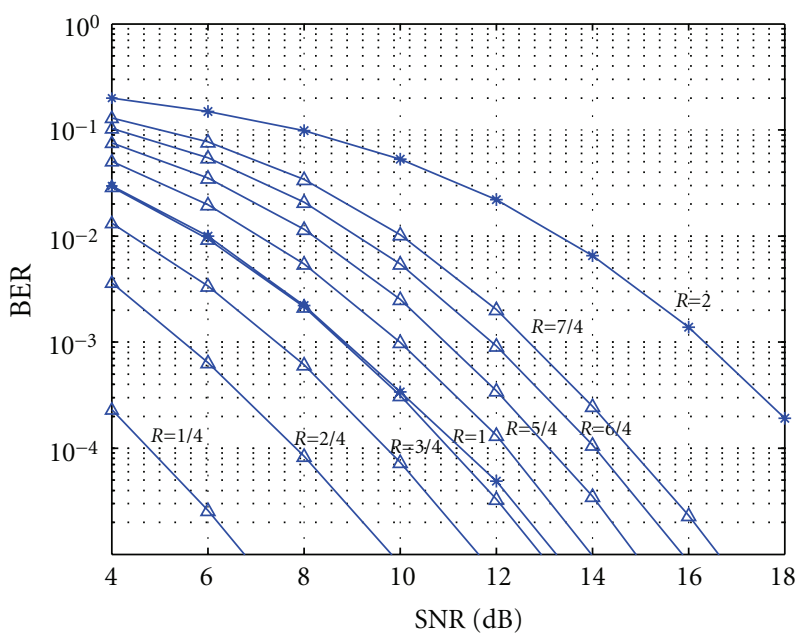

FIGURE 8: Multirate MRP simulations marked by $\Delta$ with the optimal rotation angle $\phi_{2,2}$ and the SFBC in [12] marked by $*$ for a MIMOOFDM system with $N_{t}=2, N_{r}=2, N_{c}=512, P=4$ and, $\delta=128$ in COST207 typical urban scenario.

is specified by the second column in Table 3 for a variety of transmission rates $R$.

It is shown in Figure 8 that with the decrease of transmission rate, the coding gain lowerbound $\breve{\xi}$ is increased and consequently the BER performance is better. The SNR gain is roughly $1 \mathrm{~dB}$ for each decrement of transmission rate. Hence the coding matrix (39) shows a flexible structure so that targeted BER performance can be achieved by smoothly reducing the transmission rate. Moreover, in Figure 8 the multirate SFBC is also compared with the rate one SFBC in [12] with QPSK and 16QAM constellation. The SFBC in [12] with 16QAM is compared with the multirate SFBC with $R=2$ since both SFBCs have the same spectral efficiency of $4 \mathrm{bps} / \mathrm{Hz}$. It is shown that the multirate SFBC with $R=2$ has about $4 \mathrm{~dB}$ gain. On the other hand the SFBC in [12] with 
QPSK is compared with the multirate SFBC with $R=1$ since both SFBCs have same spectral efficiency of $2 \mathrm{bps} / \mathrm{Hz}$. It is shown that the multirate SFBC with $R=1$ has about $0.2 \mathrm{~dB}$ gain of BER performance. Hence the multirate MRP achieves better spectral efficiency and a smoother balance between the transmission rate and BER performance.

\section{Conclusion}

A rate one MRP and a multirate MRP are proposed for MIMO-OFDM systems. Both MRP, are capable of achieving full transmit diversity for an arbitrary number of transmit antennas, subcarrier grouping, and subcarrier interval. Moreover, the proposed rate one MRP and multirate MRP demonstrate the feasibility of the SFBC design even if the transmit antennas do not have full knowledge of channel PDP, or if the channel PDP is dominated by a limited number of delays. Both of SFBCs have more design freedom, mitigate the requirement upon subcarrier interval and subcarrier grouping, and also overcome the potential loss of diversity for specific propagation channels. The proposed rate one MRP has a relatively simple optimization process, which can be visualized directly, while the proposed multirate MRP has better spectral efficiency and provides a relatively smooth balance between system performance and transmission rate.

\section{Acknowledgment}

The authors wish to acknowledge the support of Dr. Mansoor Shafi and Andrew Mackay during the work of this paper. This work is supported by Australia Research Council Discovery Grant DP0558865.

\section{References}

[1] S. M. Alamouti, "A simple transmit diversity technique for wireless communications," IEEE Journal on Selected Areas in Communications, vol. 16, no. 8, pp. 1451-1458, 1998.

[2] C. F. Mecklenbräuker, M. Rupp, and G. Gritsch, "On mutual information and outage for extended alamouti space-time block codes," in Proceedings of the IEEE Sensor Array and Multichannel Signal Processing Workshop (SAM '04), pp. 274278, July 2004.

[3] B. Hassibi and B. M. Hochwald, "High-rate codes that are linear in space and time," IEEE Transactions on Information Theory, vol. 48, no. 7, pp. 1804-1824, 2002.

[4] F. Oggier, G. Rekaya, J.-C. Belfiore, and E. Viterbo, "Perfect space-time block codes," IEEE Transactions on Information Theory, vol. 52, no. 9, pp. 3885-3902, 2006.

[5] WiMax, http://www.wimaxforum.org.

[6] 3GPP TR 36.913 v 8.0.1, "Requirements for further advancements for Evolved Universal Terrestrial Radio Access (EUTRA) (LTE-Advanced)," March 2009.

[7] G. T. . V8.5.1, "User Equipment (UE) radio transmission and reception for Evolved Universal Terrestrial Radio Access (EUTRA)," March 2009.

[8] W. Zhang, X.-G. Xia, and P. Ching, "High-rate full-diversity space-time-frequency codes for broadband MIMO blockfading channels," IEEE Transactions on Communications, vol. 55, no. 1, pp. 25-34, 2007.
[9] Y. Xin, Z. Wang, and G. B. Giannakis, "Space-time diversity systems based on linear constellation precoding," IEEE Transactions on Wireless Communications, vol. 2, no. 2, pp. 294-309, 2003.

[10] J.-C. Belfiore, G. Rekaya, and E. Viterbo, "The golden code: a $2 \times 2$ full-rate space-time code with nonvanishing determinants," IEEE Transactions on Information Theory, vol. 51, no. 4, pp. 1432-1436, 2005.

[11] J. Ong, A. Jayalath, and C. Athaudage, "Adaptive timefrequency spreading of quasi-orthogonal block codes for MIMO-OFDM systems," in Proceedings of the 10th IEEE Singapore International Conference on Communication Systems (ICCS '06), pp. 1-7, October 2006.

[12] L. Shao and S. Roy, "Rate-one space-frequency block codes with maximum diversity for MIMO-OFDM," IEEE Transactions on Wireless Communications, vol. 4, no. 4, pp. 1674-1686, 2005.

[13] W. Su, Z. Safar, and K. Liu, "Full-rate full-diversity spacefrequency codes with optimum coding advantage," IEEE Transactions on Information Theory, vol. 51, no. 1, pp. 229249, 2005.

[14] L. Shao, S. Roy, and S. Sandhu, "Rate-one space frequency block codes with maximum diversity gain for MIMO-OFDM," in Proceedings of the IEEE Global Telecommunications Conference (GLOBECOM '03), vol. 2, pp. 809-813, December 2003.

[15] X. Zhu and J. Xue, "On the correlation of subcarriers in grouped linear constellation precoding OFDM systems over frequency selective fading," in Proceedings of the 63rd IEEE Vehicular Technology Conference (VTC '06), vol. 3, pp. 14311435, Melbourne, Australia, May 2006.

[16] WINNER, "Final report on link level and system level channel models, d5.4 v1.4," IST-2003-507581.

[17] Z. Liu, Y. Xin, and G. B. Giannakis, "Space-time-frequency coded OFDM over frequency-selective fading channels," IEEE Transactions on Signal Processing, vol. 50, no. 10, pp. 24652476, 2002.

[18] V. Tarokh, H. Jafarkhani, and A. R. Calderbank, "Space-time block codes from orthogonal designs," IEEE Transactions on Information Theory, vol. 45, no. 5, pp. 1456-1467, 1999.

[19] C. Tepedelenlioglu, "Maximum multipath diversity with linear equalization in precoded OFDM systems," IEEE Transactions on Information Theory, vol. 50, no. 1, pp. 232-235, 2004.

[20] S. Gowrisankar and B. S. Rajan, "A rate-one full-diversity lowcomplexity space-time-frequency block code (STFBC) for 4Tx MIMOOFDM," in Proceedings of the IEEE International Symposium on Information Theory (ISIT '05), pp. 2090-2094, Adelaide, Australia, September 2005.

[21] J. Jin, K.-W. Ryu, and Y. Park, "A full rate quasi-orthogonal STF-OFDM with DAC-ZF decoder over wireless fading channels," ETRI Journal, vol. 28, no. 1, pp. 87-90, 2006.

[22] W. Su and X.-G. Xia, "Signal constellations for quasiorthogonal spacetime block codes with full diversity," IEEE Transactions on Information Theory, vol. 50, no. 10, pp. 23312347, 2004.

[23] W. Su, Z. Safar, and K. R. Liu, "Towards maximum achievable diversity in space, time, and frequency: performance analysis and code design," IEEE Transactions on Wireless Communications, vol. 4, no. 4, pp. 1847-1857, 2005.

[24] W. Zhang, X.-G. Xia, and P. Ching, "High-rate full-diversity space-time-frequency codes for MIMO multipath blockfading channels," in Proceedings of the IEEE Global Telecommunications Conference (GLOBECOM '05), vol. 3, pp. 1587-1591, November 2005. 
[25] J. Wu and S. D. Blostein, "Linear dispersion over time and frequency," in Proceedings of the IEEE International Conference on Communications (ICC '04), vol. 1, pp. 254-258, June 2004.

[26] 3GPP and 3GPP2, "Spatial channel model AHG," 5.0ed, April 2003.

[27] COST 207 Management Committee, "Digital land mobile radio communications," Final Report, Commission of the European Communities, 1989.

[28] A. F. Molisch, M. Z. Win, and J. H. Winters, "Space-timefrequency (STF) coding for MIMO-OFDM systems," IEEE Communications Letters, vol. 6, no. 9, pp. 370-372, 2002.

[29] M. Zhang, P. J. Smith, and M. Shafi, "A new space-time MIMO channel model," in Proceedings of the 6th Australian Communications Theory Workshop (AUSCTW'05), pp. 249254, February 2005.

[30] K. Yu, M. Bengtsson, B. Ottersten, D. McNamara, P. Karlsson, and M. Beach, "Modeling of wide-band MIMO radio channels based on NLoS indoor measurements," IEEE Transactions on Vehicular Technology, vol. 53, no. 3, pp. 655-665, 2004.

[31] A. K. Sadek, W. Su, and K. R. Liu, "Diversity analysis for frequency-selective MIMO-OFDM systems with general spatial and temporal correlation model," IEEE Transactions on Communications, vol. 54, no. 5, pp. 878-887, 2006.

[32] Z. Liu, Y. Xin, and G. Giannakis, "Linear constellation precoding for OFDM with maximum multipath diversity and coding gains," IEEE Transactions on Communications, vol. 51, no. 3, pp. 416-427, 2003.

[33] R. A. Horn and C. R. Johnson, Matrix Analysis, Cambridge University Press, Cambridge, UK, 1986.

[34] L. Xian and H. Liu, "Optimal rotation angles for quasiorthogonal spacetime codes with PSK modulation," IEEE Communications Letters, vol. 9, no. 8, pp. 676-678, 2005.

[35] M. Zhang, T. D. Abhayapala, D. Jayalath, D. Smith, and C. Athaudage, "Multirate space-time-frequency linear block coding," in Proceedings of the IEEE International Conference on Communications (ICC '08), pp. 4084-4089, May 2008.

[36] K. Su, "Efficient maximum likelihood detection for communication over multiple input multiple output channels," Technical Report, University of Cambridge, Cambridge, UK, February 2005, http://www.cl.cam.ac.uk/research/dtg/ publications/public/ks349/Su05B.pdf.

[37] K. Su and I. J. Wassell, "A new ordering for efficient sphere decoding," in Proceedings of the IEEE International Conference on Communications (ICC '05), vol. 3, pp. 1906-1910, May 2005. 